\title{
La construcción de escenarios utilizando un sistema de inferencia difuso para la optimización estocástica del rediseño de la cadena de suministro de reciclaje
}

\author{
The construction of scenarios using a diffuse inference system for stochastic \\ optimization of the recycling supply chain redesign \\ Michael Feitó Cespón ${ }^{1 *}$ \\ Recibido 13 de septiembre de 2018, aceptado 17 de junio de 2019 \\ Received: September 13, 2018 Accepted: June 17, 2019
}

\begin{abstract}
RESUMEN
En este artículo se traza el objetivo construir un conjunto de escenarios para su utilización en la solución de un modelo estocástico de dos etapas para el rediseño de la cadena de suministros de reciclaje de plásticos en Cuba y así minimizar el efecto de la incertidumbre en la toma de estas decisiones estratégicas. La información estadística recolectada en la caracterización de la cadena es escasa y no es suficiente para conformar escenarios a través de los métodos cuantitativos encontrados en la literatura. Para resolver esta problemática se utiliza un sistema de inferencia difuso, el cual permite la vinculación de la información estadística sobre el sistema y el conocimiento de expertos en reciclaje y gestión de la cadena de suministros de una forma robusta. Esta metodología, aplicada a la recuperación de plásticos en Cuba, posibilita aumentar el número de soluciones del modelo a su vez que, integrado al método de solución de dos etapas, evalúa las soluciones para encontrar las más prometedoras. Se obtiene un total de nueve escenarios y se procede a la optimización del modelo propuesto con el cual se obtiene un conjunto de 225 soluciones, encontrando como la mejor, para todos los escenarios, abrir tres plantas de reciclaje.
\end{abstract}

Palabras clave: Construcción de escenarios, sistema de inferencia difusa, rediseño de la cadena de suministro, logística inversa.

\begin{abstract}
This paper aims to build a set of scenarios for its use in the solution of a stochastic two-stage optimization model for the redesign of the plastics recycling supply chain in Cuba and thus minimize the effect of uncertainty in this strategic decision-making process. The statistical information collected in the characterization of the supply chain is scarce and it is not enough to conform scenarios through the quantitative methods found in the literature. To solve this problem a diffuse inference system is used, which allows the linking of statistical information about the system and the knowledge of experts in recycling and supply chain management in a robust way. This methodology, applied to the plastics recycling in Cuba, makes it possible to increase the number of solutions of the model which, integrated to the twostage solution method, evaluates the solutions to find the most promising ones. A total of nine scenarios are obtained and the optimization of the proposed model is carried out with which a set of 225 solutions is obtained, finding as the best, for all scenarios, the opening of three recycling plants.
\end{abstract}

Keywords: Construction of scenarios, fuzzy inference system, redesign of the supply chain, reverse logistics.

\footnotetext{
1 Universidad de Cienfuegos. Departamento de Ingeniería Industrial. Cienfuegos, Cuba. E-mail: mfeito@ucf.edu.cu

* Autor de correspondencia: mfeito@ucf.edu.cu
} 


\section{INTRODUCCIÓN}

La recuperación de plásticos es uno de los renglones de reciclaje de materiales que mayor auge está tomando en Cuba. Por esa razón, se han adquirido tecnologías para aprovechar las oportunidades que brinda el mercado mundial, y además para su consumo interno. La situación descrita ofrece un entorno favorable para la Empresa de Recuperación de Materias Primas (ERMP), la cual comenzó el reciclaje de plásticos introduciendo una planta de procesamiento, ubicada en la ciudad de Cienfuegos. Sin embargo, las deficiencias en el diseño de la cadena de suministro de reciclaje en la ERMP y en su gestión, conducen a la imposibilidad de aprovechar los potenciales de generación de estos residuos. El rediseño de la cadena de reciclaje de plásticos es una solución viable a los problemas encontrados en su diagnóstico [1].

Los procesos de reciclaje son estudiados por la llamada logística inversa, la cual se define como el conjunto de actividades de gestión que se ocupa de devolver los productos y materiales a los procesos productivos y mercados, con el fin de lograr ventajas competitivas, que se revaloricen estos materiales retornados y además, se reduzca su impacto en la naturaleza [2].
Uno de los problemas fundamentales que enfrentan los métodos para la toma de decisiones en la gestión de logística inversa, es al alto grado de incertidumbre en varios de sus parámetros como son: la generación de productos y materiales recuperables, la cantidad y calidad que reingresan a las cadenas de suministro, así como en la demanda, precios de los mismos una vez que son aptos nuevamente para su consumo [3-5].

Para enfrentar la optimización de problemas con incertidumbre se reconocen fundamentalmente tres áreas dentro de la investigación de operaciones: la programación estocástica [6-9], la programación robusta [10-11] y más recientemente la programación difusa $[4,12,13]$. En la Figura 1 se muestran los diferentes enfoques que han tratado la incertidumbre en el diseño de cadenas de suministro.

El primer enfoque se utiliza cuando se dispone de datos históricos confiables para parámetros de entrada inciertos, mientras que los otros dos se aplican en los casos en que no hay o hay pocos datos históricos sobre parámetros imprecisos. El enfoque de programación difusa también es capaz de utilizar el dominio de los expertos para estimar la función de membresía de parámetros inciertos. Sin embargo, los enfoques de optimización robustos usualmente usan conjuntos convexos para ilustrar

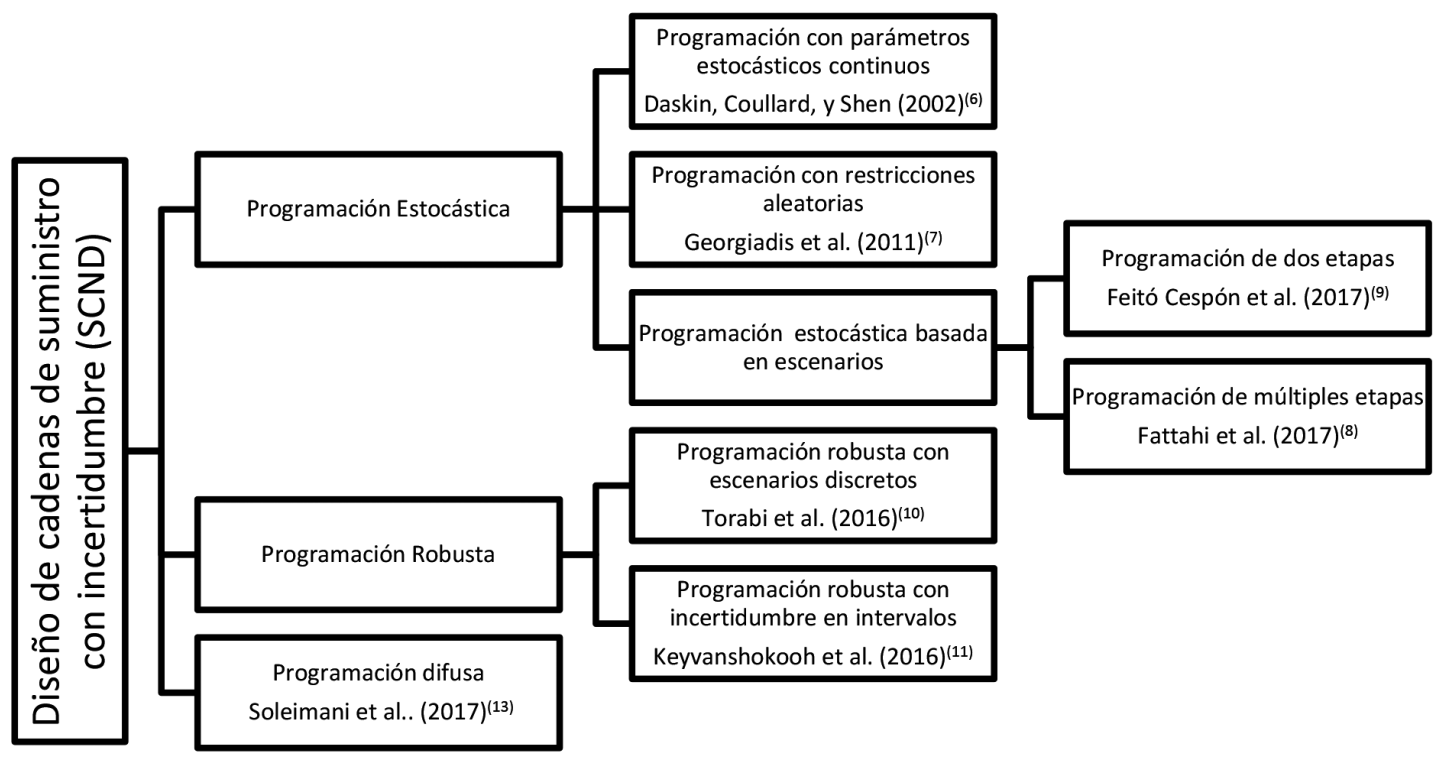

Figura 1. Métodos de optimización para el diseño de cadenas de suministro (SCND) con incertidumbre. Elaborado a partir de Govindan, Fattahi y Keyvanshokooh, (2017) [5]. 
los parámetros inciertos sin presentar ninguna información sobre la distribución de probabilidad de ellos. También hay algunos métodos que combinan los enfoques de optimización robustos y difusos [14] o métodos de optimización robustos y basados en escenarios [15] para beneficiarse de las ventajas de ambos enfoques.

Como se puede observar en la Figura 1 varios de los métodos descritos para la optimización en el diseño de la CS, ya sea a través de la programación estocástica o robusta, utilizan escenarios en su solución. Una problemática importante es la generación de estos escenarios de manera que caractericen la mayoría de los futuros posibles [16]. El problema radica en generar diferentes combinaciones de valores del conjunto de parámetros sensibles para el modelo y que sea probable su ocurrencia. Este conjunto de combinaciones intenta poseer dos características importantes: i) debe ser exhaustivo, cada combinación debe caracterizar correctamente una posibilidad y la suma de ellas debe caracterizar todo el conjunto de posibilidades; ii) la cantidad de escenarios debe poder ser manejado para el proceso de toma de decisiones, usualmente cada escenario genera una o varias optimizaciones adicionales e información que debe ser analizada por el centro de decisión, lo que requiere gastos de tiempo y recursos [5].

Los métodos para la construcción de escenarios se basan en obtener muestras, ya sea de la aproximación a la distribución probabilística subyacente del proceso estocástico del fenómeno estudiado o directamente de las series de datos; y luego se prueba que estas muestras sean suficientes para caracterizar la distribución de los datos y manejables para la toma de decisiones. En este sentido se han utilizado diversas técnicas como: algoritmos de agrupamiento [17], simulación y simulación con optimización [18-19], series de tiempo y pronóstico [20] e inteligencia artificial [21-22], entre otras.

Estos métodos son de poca utilidad cuando el proceso estocástico estudiado es relativamente nuevo y no cuenta con una base de datos histórica, o cuando se prevén comportamientos que no pueden ser descritos por estas series históricas. En estas condiciones, es importante asistirse del sentido común y la experiencia de los implicados en la toma de decisiones entre otros expertos comprometidos con los fenómenos que se estudian.
De acuerdo con lo antes expuesto, un conjunto de investigaciones se centra en el uso de técnicas cualitativas y análisis de expertos para la construcción de escenarios. En estos casos se emplean métodos participativos de grupos utilizando diversas técnicas como son: construcción de redes causales [23], visualización de historias, rueda de futuros, evaluación de narrativas [24] y el conocido método Delphi [25]. La principal deficiencia del empleo de los métodos cualitativos radica en la falta de precisión, por lo que son soportados por estadísticas que miden la concordancia y consenso.

Sin dudas, la lógica difusa y los sistemas de inferencia difusos (FIS por sus siglas en inglés), han devenido en las herramientas matemáticas que mayor impacto han tenido en la comprensión de la incertidumbre [26] y en la traducción de la experiencia humana en los sistemas complejos y la toma de decisiones [22]. El propósito de este estudio es la aplicación de los FIS en la generación de escenarios, para el uso en la optimización estocástica para el rediseño de CS sostenible de reciclaje de plásticos.

La relevancia de este artículo reside en la utilización del conocimiento de un grupo de expertos, así como la utilización de la información estadística, en la elaboración de un FIS capaz de construir escenarios utilizables en la optimización estocástica de un problema de rediseño de cadenas de suministro de reciclaje de plásticos. Este método resuelve el problema de la falta de información estadística relevante, aunque utiliza la existente vinculada a la experiencia de los directivos. El uso de la lógica difusa traduce de forma sencilla la experiencia del grupo experto para la predicción de los futuros posibles del sistema, expresado en valores numéricos necesarios para la implementación del modelo de optimización empleado.

Para facilitar la comprensión de las variables relacionadas en los escenarios y la utilización en la toma de decisiones para el rediseño de la CS de reciclaje de plásticos, primeramente se exponen el modelo multiobjetivo e indicadores de evaluación de las soluciones, luego la metodología para generar los escenarios utilizando el FIS, a continuación se presenta la aplicación de la metodología al caso de estudio y por último se presentan resultados de la aplicación del modelo estocástico resuelto para los escenarios construidos. 


\section{REVISIÓN DE LA LITERATURA}

Sin lugar a dudas la confección de escenarios ha sido sumamente utilizada no solo para los problemas de optimización, sino que se ha convertido en una poderosa herramienta para la planificación estratégica y el soporte a la toma de decisiones [27-28], un escenario se define como "La parte de la planificación estratégica que se relaciona con las herramientas y tecnologías para gestionar las incertidumbres del futuro" [29].

El uso de los escenarios para el soporte de la toma de decisiones es un tema con auge en la literatura científica [30]. En esta área del conocimiento se observan tres escuelas principales, i) lógicas intuitivas [28], ii) tendencias probabilísticas modificadas (PMT) [27], y iii) la prospectiva [30]. Los dos últimos presentan en común un enfoque en la probabilidad y la identificación de los escenarios más probables, y el uso de consultores expertos que estén familiarizados con las herramientas de modelado cuantitativo subyacentes. En contraste, el método de lógicas intuitivas es un enfoque cualitativo, basado en procesos grupales, que se enfoca en el desarrollo de múltiples escenarios que exploran los límites de posibilidad para el futuro sin tener en cuenta las cuestiones de probabilidad [28]. Estas escuelas están relacionadas, donde el principio de la lógica intuitiva, hasta cierto punto, se integra en PMT y la prospectiva y donde la perspectiva prospectiva sintetiza los principios de la PMT y luego agrega el análisis morfológico.

La mayoría de las técnicas de construcción de escenarios tienen su fundamento en métodos estadísticos [31]. Ejemplo de esto es el uso de innovaciones Gausianas y Pareto estable no Gausianas por Tokat, Rachev, y Schwartz, (2003) en problemas de inversiones [32]. Oliveira, Souza, y Marcato, (2015) constituyen un ejemplo del uso series de tiempo en la construcción de escenarios aplicado a la planeación energética [33], en este sentido Jiao, Wang, y Guo, (2014) utilizan técnicas de agrupamiento para la realización de clúster aplicado a la demanda de energía horaria, la velocidad del viento y la radiación solar para la planeación de almacenamiento de energía [34].

Un grupo importante de estudios sobre generación de escenarios utilizan diferentes métodos de optimización y simulación. Dentro de ellos Calfa et al., (2014) propone el uso de un método general de optimización general, basado en la optimización basada en data-driven para generar un árbol de escenarios aplicado un problema de planificación de producción con incertidumbre en el rendimiento de producción [20]. También se reporta el uso de técnicas de simulación y optimización y la implementación paralela, por ejemplo Beraldi et al., (2010), ha probado estas técnicas exhaustivamente en datos financieros [19].

Más recientemente las aplicaciones de BigData, Inteligencia artificial (IA) y Lógica difusa (FL) han sido estudiadas e incorporadas como herramientas potentes en la elaboración de escenarios, introduciendo un nuevo campo que se alimenta de las metodologías antes expuestas [27]. El uso de las redes neuronales y la inteligencia artificial aporta sistemas que aprenden de los errores en la construcción de escenarios. En uno de los primeros artículos que conforman este grupo, Li et al., (1997) utilizan una red neuronal de conjunto con la teoría de la verdad para la realización de árboles de escenarios utilizados en la planeación de negocios [21]. Dentro de los más recientes aportes en la generación de escenarios, se encuentra el uso de redes neuronales adaptativas para sistemas de inferencia difusas combinadas con otras herramientas fundamentalmente de optimización, con múltiples aplicaciones, ejemplo de ellas el trabajo de Araghi, Khosravi, Creighton, y Nahavandi, (2017) en sistemas de control de tráfico urbano [35] y Moayer y Bahri, (2009) en la planeación estratégica de negocios [22], por citar algunos.

Los métodos cuantitativos basados tanto en estadística como optimización y simulación requieren cantidades de datos históricos relevantes y precisos. De igual manera los métodos basados en inteligencia artificial requieren de un volumen importante de datos para lograr el aprendizaje de los sistemas generadores de escenarios. Es por esta razón que un grupo importante de trabajos refiere el uso del conocimiento de las personas, cuando el acceso a bases de datos históricas no es posible o la complejidad de las interacciones de las variables que se relacionan en la formación del escenario no permite su modelación matemática.

Se describe en la literatura un grupo importante de estudios que utilizan métodos cualitativos en 
la generación de escenarios, ejemplo de estos son: El uso de métodos de expertos relacionados con razonamiento cualitativo para la generación de modelos de biocombustible complejos [23], métodos participativos basados en redes causales y el desarrollo participativo de escenarios normativos cualitativos para elaboración de estrategias de desarrollo de generación de electricidad renovable en Alemania [36], métodos participativos como visualización de historias, rueda de futuros y evaluación de narrativas para la construcción de escenarios para la gestión energética [24], desarrollo de escenarios para futuras soluciones de transporte sostenible utilizando el método Delphi [25], y la integración de múltiples métodos cualitativos y cuantitativos en la formación de escenarios para incluir el cambio climático en la toma de decisiones [37], por citar algunos. La principal debilidad de estos métodos es la falta de precisión, aunque la mayoría utilizan estadísticas para su validación como coeficientes de concordancia o análisis de consenso.

En la Tabla 1 se muestra un resumen de las principales características de las metodologías generales de construcción de escenarios a partir de la revisión de la literatura. Uno de los problemas más importantes que se identifican en la literatura es la necesidad de conseguir grandes volúmenes de información, fundamentalmente sobre datos históricos de los fenómenos estudiados, por parte de los autores que utilizan métodos cuantitativos. Sin embargo, se afecta la precisión y la confiabilidad de aquellos que se sirven del criterio de expertos como elemento esencial. En este sentido se demuestra la necesidad de la consulta a expertos externos y partes involucradas en los fenómenos independientemente que el método utilizado sea cuantitativo, aunque las diferentes escuelas tienen una visión de su forma de involucrarse con la generación de los escenarios.

Un aspecto relevante de la construcción de los escenarios es la vinculación que tiene el método utilizado con el contexto del escenario, en cuanto a alcance, variables a utilizar, información con que se cuenta entre otras. Esta característica hace que los métodos se desarrollen en función de un problema en cuestión aunque enmarcadas en metodologías generales [16].

El artículo propuesto utiliza la información cuantitativa estadística viable y utiliza varias decisiones a partir del conocimiento de un grupo experto para la elaboración de un FIS que modela los escenarios para su utilización en la programación estocástica. Sin dudas el método utilizado permite una combinación de los datos existentes y la información aportada por los expertos de manera coherente.

Aunque generalizable la metodología para construcción de escenarios propuesta, el FIS diseñado tiene una vinculación estrecha con el problema práctico que se desea resolver (rediseñar la cadena de suministro de reciclaje de plásticos) y con la estrategia de solución del modelo de optimización multiobjetivo estocástico propuesto para su solución.

Tabla 1. Características de las metodologías para la construcción de escenarios derivada de la revisión de la literatura.

\begin{tabular}{|l|l|l|l|c|}
\hline Metodologías & \multicolumn{1}{|c|}{ Enfoque Cuantitativo } & \multicolumn{1}{|c|}{ Enfoque Cualitativo } & Información & $\begin{array}{c}\text { Referencias } \\
\text { recientes }\end{array}$ \\
\hline Lógica intuitiva & $\begin{array}{l}\text { Muy poco cuantitativo, utilizan } \\
\text { valores numéricos básicos como } \\
\text { información para los expertos }\end{array}$ & $\begin{array}{l}\text { Combina las partes interesadas } \\
\text { que aportan ideas y opiniones } \\
\text { de expertos. }\end{array}$ & $\begin{array}{l}\text { Poco demandante de volúmenes } \\
\text { altos de información confiable. }\end{array}$ & $\begin{array}{c}{[24,28,36,} \\
38-41]\end{array}$ \\
\hline PMT & $\begin{array}{l}\text { Cuantitativo, extremadamente } \\
\text { probabilístico }\end{array}$ & $\begin{array}{l}\text { Poco utilizado el criterio de } \\
\text { experto }\end{array}$ & $\begin{array}{l}\text { Demandante de volúmenes de } \\
\text { información histórica altos y } \\
\text { confiables }\end{array}$ & $\begin{array}{c}{[9,15,} \\
42-43]\end{array}$ \\
\hline Prospectiva & Cuantitativo probabilístico & $\begin{array}{l}\text { Rol de liderazgo de expertos } \\
\text { externos }\end{array}$ & $\begin{array}{l}\text { Demandante de volúmenes de } \\
\text { información histórica altos y } \\
\text { confiables }\end{array}$ & {$[44-47]$} \\
\hline $\begin{array}{l}\text { BigData, IA } \\
\text { y FL }\end{array}$ & $\begin{array}{l}\text { Cuantitativo, no necesariamente } \\
\text { probabilístico }\end{array}$ & $\begin{array}{l}\text { Rol de liderazgo de expertos } \\
\text { externos }\end{array}$ & $\begin{array}{l}\text { Demandante de grandes volú- } \\
\text { menes de información aunque } \\
\text { con menos demanda de certeza }\end{array}$ & $\begin{array}{c}{[15,22,} \\
48-51]\end{array}$ \\
\hline
\end{tabular}




\section{MODELO MULTIOBJETIVO ESTOCÁSTICO PARA EL REDISEÑO DE LA CS SOSTENIBLE PARA EL RECICLAJE DE MÚLTIPLES PRODUCTOS}

El problema de diseño de la cadena de suministro para el reciclaje consiste en determinar los centros de recolección y las plantas de procesamiento, así como las necesidades de transporte que permita cumplir con la demanda, optimizando los costos de operaciones y la utilización de las capacidades. El rediseño comprende decisiones similares partiendo de una red logística existente y con el supuesto de que la CS rediseñada debe posibilitar la mejora de su desempeño. Para el caso del reciclaje de múltiples productos este problema se formaliza a continuación:

\section{Sean:}

- Los h tipos de instalaciones diferentes necesarias para obtener, recolectar, procesar y reutilizar materiales, conocidas las posibles localizaciones de suministradores, centros de recolección, plantas de procesamiento y clientes ( $i, j, k$ y 1 respectivamente), los medios de transporte $\mathrm{m}$ y sus capacidades, en condiciones de incertidumbre de generación de residuos y demanda de materiales.

- Determinar, la organización del flujo de los p materiales diferentes Qp, la cantidad y ubicación de las instalaciones, la cantidad de viajes y tipo de medios de transporte necesarios que:

- Min f1(Qp): minimice el costo total del sistema para reciclar y vender los materiales;

- Max f2(Qp): maximice el ahorro en el impacto ambiental del sistema por utilizar materiales reciclados; y Max f3(Qp): maximice el flujo al cliente.

El tratamiento al problema del rediseño debe considerar que cualquier solución obtenida a través del modelo permita un desempeño superior al existente en la cadena actual. Este problema puede ser resuelto a partir de comparar los resultados de las funciones objetivo con los valores actuales que caracterizan el comportamiento de la CS que se rediseña. Sin embargo, este propósito resulta más complejo cuando existe incertidumbre en la determinación de algunos parámetros. Para este caso sería necesario obtener una configuración de la red que mantenga un desempeño sostenible, aun cuando se prevean cambios importantes en la generación y la demanda de materiales reciclables, por lo que los resultados del modelo deben poder compararse con proyecciones del desempeño de la $\mathrm{CS}$ actual para estas condiciones diferentes.

El modelo multiobjetivo para el rediseño de la CS de reciclaje se encuentra descrito en el Anexo 1 $[9,52]$. La estrategia para construir el conjunto de soluciones Pareto-óptimas para este problema, según el método de las restricciones se puede describir como:

$$
\begin{gathered}
\min f_{1},(\mathrm{~A} 1) \text { Anexo } 1 \\
s, t, f_{2}(x) \leq f_{2}^{\min }(x)+k \Delta \varepsilon_{2},(\mathrm{~A} 2) \text { Anexo } 1 \\
f_{3}(x) \leq_{2}^{\min }(x)+k \Delta \varepsilon_{3},(\mathrm{~A} 3) \text { Anexo } 1 \\
\text { Restricciones }(\mathrm{A} 4)-(\mathrm{A} 17) \text { Anexo } 1 \\
\text { con } k=1, \ldots, n \text { y } \Delta \varepsilon_{i}=\frac{f_{i}^{\max }-f_{i}^{\min }}{n}, i=23
\end{gathered}
$$

Resultado de este proceso se obtiene una frontera de Pareto de diferentes configuraciones de la CS, el problema reside en determinar cuáles de estas soluciones son robustas a los cambios de generación y demanda de los materiales reciclados que pueden ocurrir en el futuro. Desde ese punto de vista, resulta necesario desarrollar un indicador que evalúe integralmente los aspectos contenidos en las funciones objetivo y que, a efectos de la programación robusta, signifique una medida de la factibilidad de la solución. Generalmente esta es una función financiera que mide el riesgo de la inversión ante diferentes condiciones [53-55].

En este artículo se propone un indicador multicriterio que represente la posibilidad de una solución de desarrollar el sistema sosteniblemente. Este indicador se ha denominado potencial de la solución y se define como el máximo nivel de servicio (NSC) que puede alcanzar una configuración de la cadena, y que no empeore un determinado desempeño económico y ecológico, teniendo en cuenta escenarios donde varían las generaciones y demandas de los materiales reciclados.

El NSC es la probabilidad de cumplir con determinado requerimiento de los clientes como las cantidades demandadas y las cantidades de pedidos servidos, 
entre otras [56]. La función objetivo que determina el flujo servido a los clientes, puede ser utilizada para calcular el NSC que permite una configuración de la CS para un determinado nivel de generación y demanda de los productos recuperados, como se muestra en la ecuación 2. La demanda es una de las variables aleatorias y está explícita en la ecuación para determinar el NSC. La generación de los residuos puede constituir una restricción para alcanzar este objetivo, por lo que, para diferentes combinaciones de estas variables, pueden existir diferentes valores de NSC. El otro aspecto que puede limitar un valor alto de este indicador es la configuración de la cadena, por lo que la ecuación 2 sería una medida apropiada para evaluar el desempeño de una configuración de la CS bajo diferentes circunstancias. La idea principal es utilizar el NSC como una medida del potencial de cada solución, calculando el valor esperado de este indicador para los diferentes escenarios a través de la ecuación 3.

$$
N S C_{i j}=\frac{f_{3 i j}}{\sum_{l} \sum_{p} D_{l p j}}
$$

Donde:

$N S C_{i j}$ es el nivel de servicio al cliente expresado en la probabilidad de cumplir con las cantidades en $\mathrm{kg}$ demandadas por los clientes, para una solución i y un escenario de generación y demanda j. $D_{l p j}$ es el valor de la demanda de cada cliente 1 del producto $\mathrm{p}$ para un escenario j y $f_{3 i j}$ es el valor de la función objetivo 3 calculada para la solución y el escenario $\mathrm{j}$.

$$
E\left(N S C_{i}\right)=\sum_{j} D C_{i j} D A_{i j} p b_{j} N S C_{i j}
$$

Donde:

$E\left(N S C_{i}\right)$ es el nivel de servicio esperado para una solución $\mathrm{i}, \mathrm{pb}_{\mathrm{j}}$ es la probabilidad de ocurrencia del escenario j y $D C_{i j}$ y $D A_{i j}$ constituyen variables binarias que representan el estado del desempeño económico y ambiental respectivamente y se definen como:

$$
\begin{aligned}
& D C_{i j}=\left\{\begin{array}{rr}
1, & \operatorname{Si} f_{1 \mathrm{ij}} \varphi_{1 \mathrm{j}}, \\
0, & \text { de cualquier otra forma. }
\end{array}\right. \\
& D A_{i j}=\left\{\begin{array}{lr}
1, & \operatorname{Si} f_{2 \mathrm{ij}} \varphi_{2 \mathrm{j}}, \\
0, & \text { de cualquier otra forma. }
\end{array}\right.
\end{aligned}
$$

Este estado muestra la condición de que la configuración obtenida en una solución nunca deteriore el desempeño actual de la CS, donde $f_{1 i j}$ y $f_{1 i j}$ son los valores de las funciones objetivo costo de operaciones y ahorro del impacto ambiental respectivamente, de una solución i para un escenario j; y los parámetros $\varphi_{1 \mathrm{j}}$ y $\varphi_{1 \mathrm{j}}$ son los valores límites de cada función. Para el caso de rediseño de la cadena de reciclaje, la determinación de estos valores está estrechamente ligada a los resultados de una caracterización económica y ecológica de la CS [1]; proyectados para los diferentes escenarios.

Para determinar el potencial de la solución es necesario volver a realizar optimizaciones del modelo para cada escenario de la manera siguiente:

\section{Para cada solución $i ; y$} para cada escenario $j$ : actualizar las restricciones, ecuaciones (A4-A17) Anexo 1; optimizar:

Max $f_{3}$ ecuación (A3) Anexo 1 sujeto a: restricción del modelo ecuaciones (A4-A17) Anexo 1

$f_{1} \leq \varphi_{1 j}$ ecuación (A1) Anexo 1

$f_{2} \geq \varphi_{2 j}$ ecuación (A2) Anexo 1; calcular NSC $C_{i j}$ : ecuación (2); calcular $E\left(N S C_{i}\right)$ : ecuación (3).

Cada solución obtenida está constituida por decisiones de capacidad, localización y asignación de las instalaciones y cada escenario brinda una combinación diferente de generación y demanda. Tanto las soluciones como los escenarios limitan el desempeño de la CS, por lo que la determinación del potencial de cada solución a través de la doble optimización, requiere de la actualización de las restricciones del modelo inicial. Este es el proceso de convertir la configuración de la cadena, brindada por una solución del modelo, en restricción para optimizar el nivel de servicio. Otro aspecto importante es la actualización de la generación en los suministradores y de la demanda en los clientes para el escenario correspondiente.

En función del indicador propuesto, es necesario que los escenarios caractericen no solo los diferentes estados de generación y demanda de plásticos reciclables y su probabilidad de ocurrencia, sino también, la 
proyección del desempeño actual de la cadena a partir del costo de operaciones y el impacto ambiental. Sin embargo, las interacciones entre estas variables no se limitan solamente a determinadas funciones, sino que la generación y demanda constituyen a su vez restricciones del sistema, lo cual complejiza el proceso de obtención de los escenarios utilizando los métodos estadísticos. Una mejor comprensión del uso del indicador así como del modelo de optimización estocástico se encuentra publicado en Feitó-Cespón, Sarache, Piedra-Jimenez y Cespón-Castro (2017) [9].

\section{METODOLOGÍA PARA LA CONSTRUCCIÓN DE LOS ESCENARIOS DE LA CS DE RECICLAJE DE PLÁSTICOS, UTILIZANDO UN SISTEMA DE INFERENCIA DIFUSO}

Para una mejor comprensión de la incertidumbre presente en la previsión de la demanda y de la generación de los materiales reciclados, en la Figura 2 se muestra el caso de la recuperación de residuos, donde el eje horizontal representa el porcentaje del potencial que la cadena recicla actualmente en un año. El eje vertical constituye la densidad de ocurrencia de esa recuperación, dado que la misma posee un comportamiento estocástico y las distribuciones mostradas serían los posibles escenarios que se pueden presentar. Una situación similar sucede con la demanda del material reciclado $y$, en consecuencia, con el costo de operaciones y el impacto ambiental, los cuales están en función de la generación y la demanda.

Partiendo de lo anterior, en el rediseño de las cadenas de reciclaje, es posible contar con la distribución de un escenario actual, representada en la Figura 2 mediante líneas continuas. Además, se puede estimar un potencial máximo obtenido por un grupo de trabajo a partir de información histórica, estudios del mercado, entre otras fuentes, aunque no deja de ser de naturaleza incierta. Esta incertidumbre puede ser formulada, empleando el concepto de los conjuntos difusos o borrosos [57]. La utilización del sistema de inferencia difusa permite al grupo de trabajo construir escenarios de forma más intuitiva, para que el modelo encuentre soluciones sobre la actualidad y los futuros probables.

La inferencia difusa, es el proceso de formular el mapeo de un conjunto de variables de entrada, en un conjunto de variables de salida, utilizando la lógica difusa. Este sistema contiene dos tipos importantes de información y su selección correcta constituye un paso crítico en el proceso de diseño del sistema, pudiendo afectar dramáticamente su desempeño. Estas son:

i. Una base de datos que define el número, etiquetas y tipos de funciones de membrecía, que el conjunto borroso utiliza como valores de cada sistema de variables lingüísticas. Esta contiene dos tipos de variables: de entrada y de salida. Para cada una de las variables se define un conjunto borroso, donde los valores que puede tomar una variable constituyen su universo del discurso.

ii. Una base de reglas lógicas, las cuales esencialmente convierten los valores de entrada en valores de salida, por lo que refleja la política de toma de decisiones del sistema. La estrategia de control se encuentra almacenada en la base de reglas, las cuales incluyen ponderaciones y combinaciones de los conjuntos difusos.

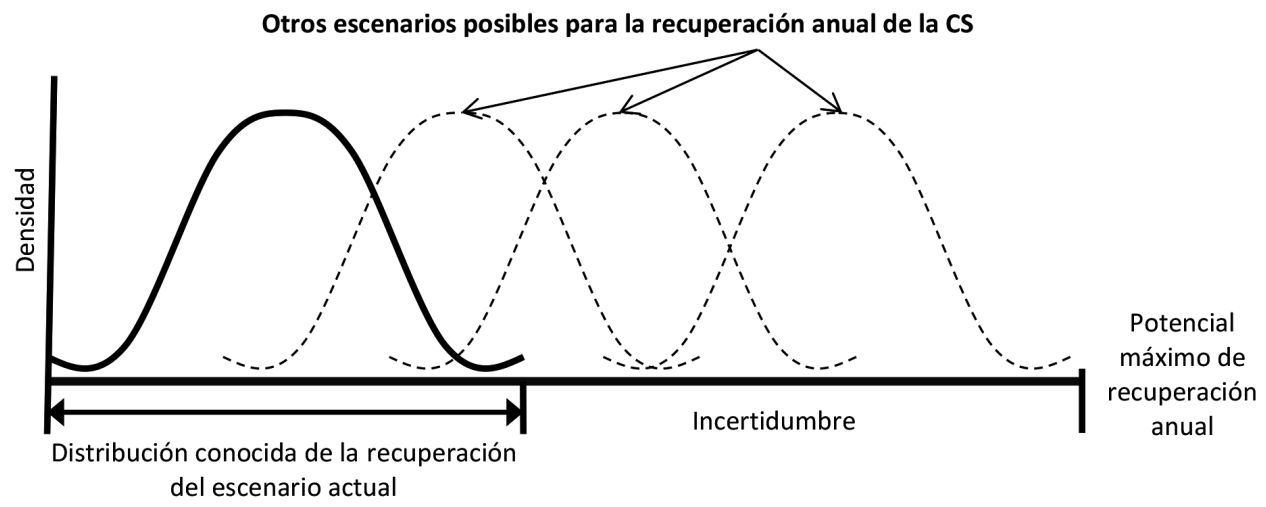

Figura 2. Representación de los escenarios para la recuperación de materiales reciclables. 
El método de inferencia difusa de Mamdani es la metodología más común desde los primeros sistemas de control que utilizaron la teoría de los conjuntos difusos. Esta fue propuesta en 1975 por Ebrahim Mamdani [58-59] para controlar un sistema de calderas y motores de vapor, sintetizando un conjunto de reglas basados en las experiencias de los operadores y tuvo su punto de partida en el trabajo de Lotfi Zadeh [60] sobre algoritmos difusos para sistemas complejos y procesos de decisión.

Los procesos de inferencia difusa están compuestos por cinco fases: i) la codificación de las variables de entrada en variables difusas; ii) la aplicación de los operadores difusos " $\mathrm{Y}$ " $\mathrm{y}$ "O $\mathrm{O}$ " en el antecedente de las reglas; iii) la obtención de los consecuentes a partir de los antecedentes; iv) la agregación de los consecuentes a través de las reglas; y v) la decodificación de las variables de salida en valores reales.

En la presente investigación se pretende modelar las combinaciones de las variables de entrada (generación y demanda de materiales reciclados), para así obtener inferencias sobre los parámetros costo de operaciones máximo $(\varphi 1)$, ahorro ambiental mínimo $(\varphi 2)$, y la probabilidad $(p b)$ de ocurrencia de estos escenarios. Si los parámetros generación $G=\sum i \sum p G_{i p}$ y demanda $D=\sum_{l} \sum_{p} G_{i p}$ se pueden definir como conjuntos difusos $\widetilde{G}$ y $\widetilde{D}$ y las variables de decisión del modelo se encuentran restringidas por dichos parámetros, las medidas para comparar las funciones objetivo en diferentes escenarios y su probabilidad de ocurrencia, constituyen también conjuntos difusos. Estos pueden ser denominados $\widetilde{Q 1}, \widetilde{Q 2}$ y $\widetilde{p b}$, y ser determinados a partir de reglas establecidas para diferentes combinaciones de $\widetilde{G}$ y $\widetilde{D}$.

Para la confección del sistema de inferencia difuso se trabajó con el software MATLAB, el cual brinda un conjunto de herramientas programables e interactivas implementadas en la librería Fuzzy Logic Toolbox, las cuales facilitan la comprensión y utilización de estos métodos.

\section{Definición de las variables lingüísticas de entrada y de salida del sistema de inferencia difuso para la conformación de escenarios}

La información disponible para la elaboración de los conjuntos difusos, es brindada por la caracterización de la CS [1], e interpretada por el grupo de trabajo a partir del pasado reciente y el potencial del sistema. Los conjuntos difusos $\widetilde{G}, \widetilde{D}, \widetilde{\varphi} 1$ y $\widetilde{\varphi} 2$ se definen como variables lingüísticas con tres estados: Bajo, Medio y Alto, con funciones de membrecía gaussianas para la pertenencia de los valores de la variable a los estados definidos. La función de membrecía gaussiana se selecciona fundamentalmente por la naturaleza continua y la asunción de normalidad de las variables involucradas en el sistema, la ecuación 4 describe su comportamiento.

$$
E\left(N S C_{i}\right)=\sum_{j} D C_{i j} D A_{i j} p b_{j} N S C_{i j}
$$

Donde: $\mu$ es la función de membrecía de los conjuntos difusos con parámetros $\sigma=1 / 6$ del valor del rango debido a que aproximadamente la campana de Gauss para la distribución normal tiene tres veces este valor a la derecha y a la izquierda del centro, así se pueden definir los rangos de tres estados que correspondan a todo el universo del discurso y c representa los valores que definen cada estado de la variable lingüística.

Los valores mínimos y máximos se determinan utilizando los valores actuales de la distribución de la generación y la demanda, el potencial de estas y un nivel límite deseado del costo de operaciones y del impacto ambiental. A partir de esta información se pueden construir las funciones de membrecía para cada conjunto difuso como se muestra en la Tabla 2.

Para el caso de la probabilidad de ocurrencia del escenario se definen igualmente tres términos lingüísticos: Baja, Media y Alta. El grupo de trabajo decide cómo valorar la probabilidad de ocurrencia de cada combinación de las variables generación y demanda a partir de su experiencia para conformar los escenarios.

\section{Definición de las reglas del sistema de inferencia difuso}

Una vez obtenidos los conjuntos difusos y sus funciones de membrecía es necesario definir un conjunto de reglas que relacionen las variables de entrada con las de salida, a través de los operadores lógicos "Y" y "O". Estas reglas deben expresar el comportamiento de la CS para todas las posibles combinaciones de valores de las variables de entrada. Su definición se realiza a partir de la intuición 
Tabla 2. Variables lingüísticas del sistema de inferencia difuso.

\begin{tabular}{|c|c|c|c|c|c|}
\hline Variable & $\begin{array}{l}\text { Tipo de } \\
\text { Variable }\end{array}$ & $\mathbf{N}$ & $\mathbf{T}$ & M & $\mathbf{U}$ \\
\hline Generación de materiales reciclables $(\mathrm{kg})$ & Entrada & G & Baja, Media y Alta & $\begin{array}{l}\mu_{\text {Gaussiana }}(x, c, \sigma) \\
\sigma=1 / 6 \text { rango } \\
c=(\min , 1 / 2 \text { rango, } \max )\end{array}$ & [min; $\max ]$ \\
\hline Demanda de materiales reciclables $(\mathrm{kg})$ & Entrada & $\mathrm{D}$ & Baja, Media y Alta & $\begin{array}{l}\mu_{\text {Gaussiana }}(\mathrm{x}, \mathrm{c}, \sigma) \\
\sigma=1 / 6 \text { rango } \\
\mathrm{c}=(\min , 1 / 2 \text { rango, } \max )\end{array}$ & [min; $\max ]$ \\
\hline Límite para el costo de operaciones (\$) & Salida & $\phi 1$ & Bajo, Medio y Alto & $\begin{array}{l}\mu_{\text {Gaussiana }}(x, c, \sigma) \\
\sigma=1 / 6 \text { rango } \\
c=(\min , 1 / 2 \text { rango, } \max )\end{array}$ & [min; $\max ]$ \\
\hline $\begin{array}{l}\text { Límite para el ahorro ambiental } \\
\text { (puntos) Ecoindicador } 99\end{array}$ & Salida & $\phi 2$ & Bajo, Medio, y Alto & $\begin{array}{l}\mu_{\text {Gaussiana }}(\mathrm{x}, \mathrm{c}, \sigma) \\
\sigma=1 / 6 \text { rango } \\
\mathrm{c}=(\min , 1 / 2 \text { rango, } \max )\end{array}$ & [min; $\max ]$ \\
\hline $\begin{array}{l}\text { Probabilidad de ocurrencia del } \\
\text { escenario }\end{array}$ & Salida & $\mathrm{pb}$ & Baja, Media y Alta & $\begin{array}{l}\mu_{\text {Gaussiana }}(\mathrm{x}, \mathrm{c}, \sigma) \\
\sigma \text { y c se definen por el } \\
\text { grupo de trabajo. }\end{array}$ & {$[0 ; 1]$} \\
\hline
\end{tabular}

* N - Nombre de la variable, T- Conjunto de términos lingüísticos, M - Conjunto de reglas semánticas que asigna a cada término su significado, y U- Universo o dominio de la variable.

y experiencia del grupo de trabajo, utilizando expresiones lógicas de:

SI Generación = Término lingüístico $\mathbf{Y}$ Demanda $=$ Término lingüístico ENTONCES Costo de operaciones = Término lingüístico; Ahorro del impacto ambiental = Término lingüístico; Probabilidad de ocurrencia del escenario $=$ Término lingüístico.

\section{Generación de las variables aleatorias}

Una vez obtenido todo el conjunto de reglas que definen las relaciones entre las variables de entrada y salida del sistema difuso, se procede a generar los valores de entrada aleatoriamente para cada uno de los escenarios. Para lograrlo, es necesario determinar la aproximación de las muestras tomadas de la práctica a las distribuciones probabilísticas conocidas, a través de la prueba no paramétrica Kolmogorov-Smirnov, para así hacer inferencias y obtener valores de posibles futuros.

Los valores tomados para la optimización del modelo son la media de la generación y de la demanda obtenida de la caracterización, expresada en la unidad de tiempo decidida. A partir de esta información, unida al potencial de la generación y la demanda, se generan los valores aleatorios que describirán las entradas del sistema de inferencia difuso. El grupo de trabajo determina las medias y los rangos que van a ser utilizados en la generación de las variables aleatorias para cada uno de los tres términos lingüísticos, cada vez que se forme una combinación de ellas en la conformación de los escenarios.

\section{CONSTRUCCIÓN DE LOS ESCENARIOS UTILIZANDO SISTEMAS DE INFERENCIA DIFUSOS. CASO DE ESTUDIO CADENA DE RECICLAJE DE PLÁSTICOS}

Debido a la incertidumbre en la predicción de la generación de los plásticos reciclables y la demanda de las empresas nacionales y la exportación de estas materias primas, es necesaria la modelación de varios escenarios posibles. Para esto, se construyó un sistema de inferencia difuso, utilizando el software profesional MATLAB. Como se muestra en la Figura 3, este sistema está comprendido por cinco variables lingüísticas, dos de entrada que representan la generación y demanda totales de plásticos reciclados $G$ y $D$ y tres de salida: $\widetilde{\varphi} 1$ que representa el costo máximo, $\widetilde{\varphi 2}$ el impacto ambiental mínimo ahorrado por reciclar los materiales en conjunto y $\widetilde{p b}$, la probabilidad de ocurrencia de un escenario.

La información de partida para conformar el sistema de inferencia difuso está constituida por 


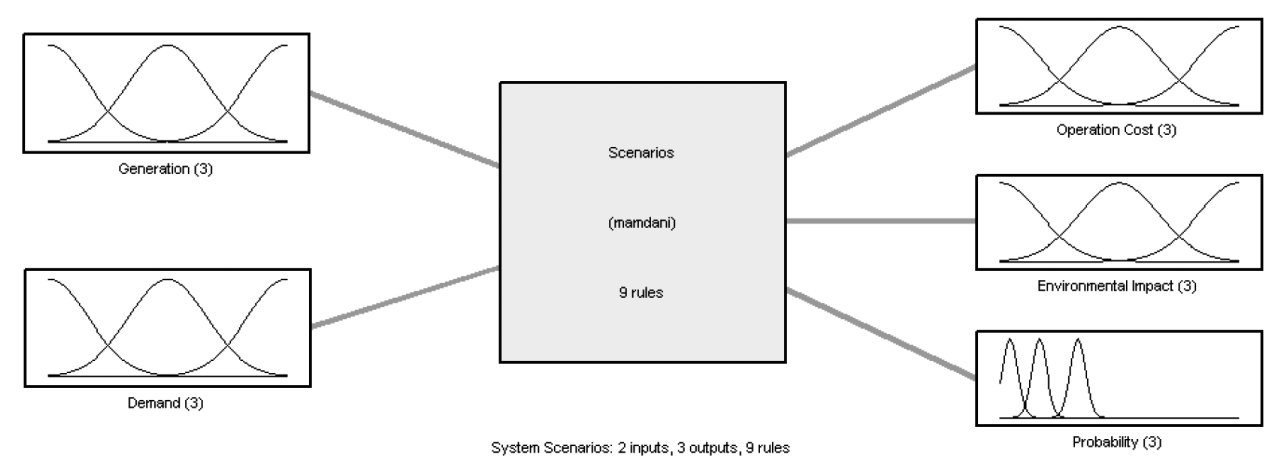

Figura 3. Esquema del sistema de inferencia difusa para la conformación de los escenarios.

las cantidades de plásticos generadas y demandadas mensualmente de cada producto. Estas constituyen variables aleatorias que se pueden aproximar a distribuciones normales, para lo cual se empleó la prueba no paramétrica de Kolmogorov-Smirnov. Los resultados presentan valores de significación asintótica mayores a 0,05 para cada variable, por lo que no se puede rechazar la idea de que provienen de una distribución Normal para un nivel de confianza del $95 \%$.

Luego de probar la normalidad de las variables generación y demanda mensuales para cada producto, se realizaron combinaciones lineales de las mismas, pues ello permite determinar sus distribuciones agregadas, modificar el horizonte de tiempo y calcular las medias y desviaciones del costo de operaciones y del impacto ambiental (ver Tabla 3 y Tabla 4).
Los datos utilizados para la construcción de los escenarios se muestran en la Tabla 4, donde se observan los valores de la media y las desviaciones de la recuperación, de la demanda, de los costos de operaciones y del impacto ambiental anual. Cabe aclarar que, a los efectos de la investigación para proyectar la demanda, se tomaron como referencia los datos de las ventas del 2013. Según el criterio del grupo de trabajo, el nivel actual de operaciones de la cadena representa aproximadamente el 30\% del potencial de generación de plásticos reciclables y su demanda en el país.

En la Tabla 4, el nivel mínimo corresponde al valor de la distribución normal de cada variable para una probabilidad de 0,001. El nivel máximo se determinó, dividiendo a la media por 0,3 , debido que los expertos consideran que en el estado actual

Tabla 3. Resumen de las estadísticas descriptivas de la recuperación y las ventas de plásticos mensuales y anuales.

\begin{tabular}{|c|c|c|c|c|c|}
\hline & & \multicolumn{2}{|c|}{ Estadística mensual } & \multicolumn{2}{|c|}{ Estadística anual } \\
\hline & & Media & Desviación & Media & Desviación \\
\hline \multirow{4}{*}{ Recuperación toneladas } & PET & 31,82 & 5,95 & 381,86 & 20,60 \\
\hline & PEL & 5,73 & 4,83 & 68,79 & 16,74 \\
\hline & MIXTO & 97,03 & 53,51 & 1164,36 & 185,38 \\
\hline & Total & 134,58 & 54,06 & 1615,00 & 187,27 \\
\hline \multirow{4}{*}{ Ventas toneladas } & PET & 15,28 & 4,82 & 183,35 & 16,69 \\
\hline & PEL & 17,13 & 5,47 & 205,54 & 18,96 \\
\hline & MIXTO & 49,67 & 10,32 & 595,99 & 35,75 \\
\hline & Total & 82,07 & 12,64 & 984,88 & 43,77 \\
\hline
\end{tabular}

* PET: polietileno tereftalato, PEL: polietileno de alta y baja densidad, MIXTO: mezcla de plásticos de diferentes tipos. 
Tabla 4. Estadísticas para las variables utilizadas en el sistema de inferencia difusa para la conformación de los escenarios.

\begin{tabular}{|l|c|c|c|c|}
\cline { 2 - 5 } \multicolumn{1}{c|}{} & Media & Desviación & Max & Min \\
\hline Generación (t/año) & $1,62 \mathrm{E}+03$ & $1,87 \mathrm{E}+02$ & $7,58 \mathrm{E}+03$ & $1,04 \mathrm{E}+03$ \\
\hline Demanda (t/año) & $9,85 \mathrm{E}+02$ & $4,38 \mathrm{E}+01$ & $4,40 \mathrm{E}+03$ & $8,50 \mathrm{E}+02$ \\
\hline Impacto Ambiental (Puntos/año) & $3,61 \mathrm{E}+05$ & $1,93 \mathrm{E}+03$ & $1,57 \mathrm{E}+06$ & $3,55 \mathrm{E}+05$ \\
\hline Costo Operaciones (Pesos/año) & $8,32 \mathrm{E}+05$ & $1,25 \mathrm{E}+03$ & $3,61 \mathrm{E}+06$ & $8,28 \mathrm{E}+05$ \\
\hline
\end{tabular}

se recupera aproximadamente un $30 \%$ del potencial, y sumándole el valor de la distribución normal para una probabilidad de 0,999 . Las medias y desviaciones totales, se formaron a partir de combinaciones lineales de las distribuciones normales de las variables de recuperación y ventas mensuales de cada tipo de plásticos, el costo de operaciones y el impacto ambiental se calculan utilizando los datos de la caracterización a través de las ecuaciones A1 y A2 descritas en el Anexo 1.

\section{Conformación de las funciones de pertenencia de las variables}

En correspondencia con la metodología descrita y con la información de la Tabla 4, se modelaron las funciones de pertenencia de cada una de estas cuatro variables. En la Figura 4a se presenta la función de pertenencia gaussiana de la generación
$\widetilde{G}$, la cual tiene tres estados representados por los términos lingüísticos Alto, Medio y Bajo. De la misma manera, se modelaron las funciones de pertenencia de la demanda (Figura 4b) el costo de operaciones (Figura 4c) y el impacto ambiental (Figura 4d).

En la Tabla 5 se describe la modelación de los posibles escenarios correspondientes a las combinaciones de los estados de la generación y la demanda, para determinar la probabilidad asociada a cada uno y conformar su función de pertenencia. El grupo de trabajo decidió valorar la ocurrencia de un estado de la variable al doble del estado más cercano, por tanto, la probabilidad de que la generación o la demanda sea la más probable es dos veces mayor que la medianamente probable, la cual es, dos veces mayor a que adquiera un valor poco probable.
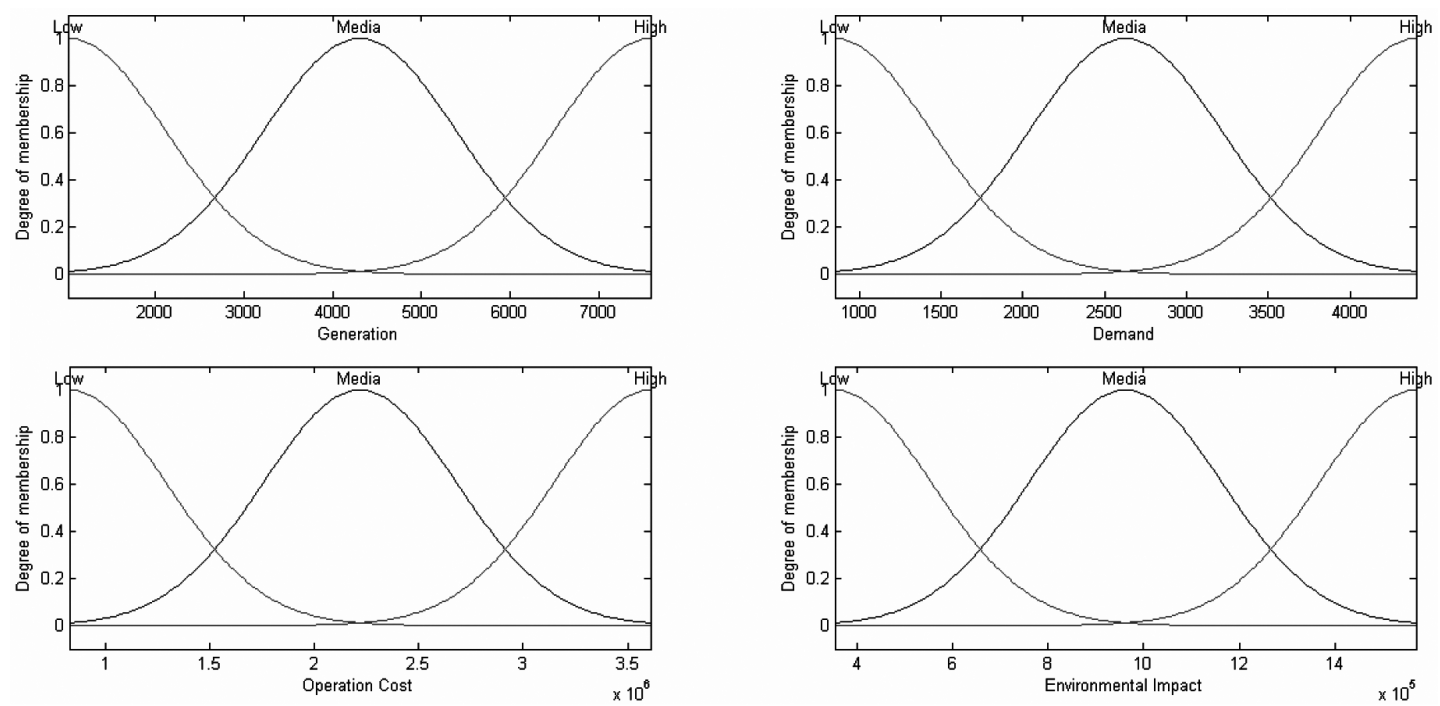

Figura 4. Funciones de pertenencia gaussianas de las variables involucradas en el SID: a) Superior Izquierda, generación de plásticos reciclables $(\mathrm{t})$, superior derecha, demanda de plásticos reciclados (t), inferior izquierda, costo de operaciones $(\$)$ e inferior derecha, impacto ambiental (Puntos). 
Tabla 5. Determinación de la probabilidad de los escenarios para la conformación del sistema de inferencia difusa.

\begin{tabular}{|c|l|c|c|c|c|}
\hline \multicolumn{2}{|c|}{ Escenario } & $\begin{array}{c}\text { Valor de ocurrencia } \\
\text { de la Generación }\end{array}$ & $\begin{array}{c}\text { Valor de ocurrencia } \\
\text { de la Demanda }\end{array}$ & pbj & $\begin{array}{c}\text { Términos } \\
\text { lingüísticos }\end{array}$ \\
\hline 1 & $\begin{array}{l}\text { Generación más probable - } \\
\text { Demanda más probable }\end{array}$ & 4 & 2 & 0.32653061 & Alta \\
\hline 2 & $\begin{array}{l}\text { Generación más probable - } \\
\text { Demanda media }\end{array}$ & 4 & 1 & 0.16326531 & Media \\
\hline 3 & $\begin{array}{l}\text { Generación más probable - } \\
\text { Demanda poco probable }\end{array}$ & 4 & 4 & 0.16326531 & Media \\
\hline 4 & $\begin{array}{l}\text { Generación media - Demanda } \\
\text { más probable }\end{array}$ & 2 & 2 & 0.08163265 & Media \\
\hline 5 & $\begin{array}{l}\text { Generación media - Demanda } \\
\text { media }\end{array}$ & 2 & 4 & 0.04081633 & Baja \\
\hline 6 & $\begin{array}{l}\text { Generación media - Demanda } \\
\text { poco probable }\end{array}$ & 2 & 2 & 0.08163265 & Media \\
\hline 7 & $\begin{array}{l}\text { Generación poco probable - } \\
\text { Demanda más probable }\end{array}$ & 1 & 1 & 0.02040816 & Baja \\
\hline 8 & $\begin{array}{l}\text { Generación poco probable }- \\
\text { Demanda media }\end{array}$ & 1 & 4 & Baja \\
\hline 9 & $\begin{array}{l}\text { Generación poco probable }- \\
\text { Demanda poco probable }\end{array}$ & 1 & 4633 & Media \\
\hline
\end{tabular}

Según el criterio de los expertos, los escenarios más probables son aquellos en que se mantengan los niveles de actividad actuales, $30 \%$ del potencial, es medianamente probable que crezcan hasta aproximadamente un $50 \%$ y poco probable que sucedan valores de generación y demanda superiores al $80 \%$ del potencial. Para el cálculo de las probabilidades que conformaron la variable lingüística se le otorgó valor de 1 a que la generación o la demanda fueran poco probable, 2 medianamente probable y 4 altamente probable, se sumaron todas las posibles combinaciones, las cuales, a su vez, se dividieron entre la suma total de ellas.
La Figura 5 representa la función de pertenencia gaussiana de la probabilidad de ocurrencia de los escenarios; de manera similar al resto de las variables difusas, presenta tres términos lingüísticos: Alto, Medio y Bajo, aunque difiere en que no es centrada como las otras. Los valores para considerar una probabilidad baja o media se encuentran más cercanos entre sí que para que sea considerada alta.

\section{Confección del sistema de reglas}

Para conectar las variables de entrada con las de salida del sistema de inferencia difuso se utilizaron nueve reglas de Si-Entonces, que describen los nueve

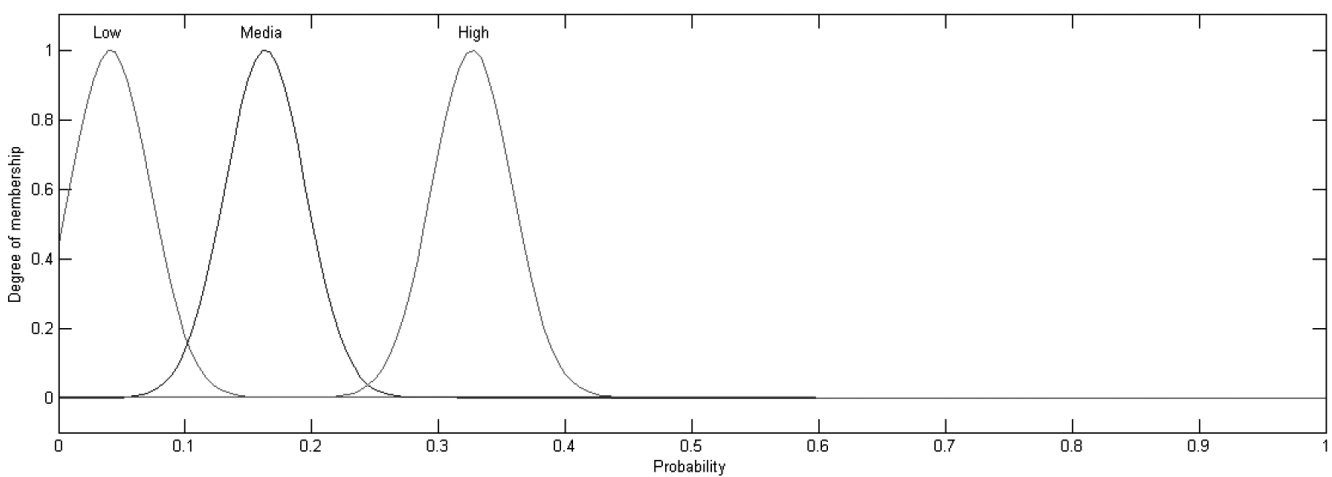

Figura 5. Función de pertenencia gaussiana de la probabilidad de ocurrencia de los escenarios. 
escenarios posibles, de las diferentes combinaciones de los estados de la generación y la demanda. Como cualquiera de estas dos variables condiciona el desempeño de la cadena, las variables de costo de operaciones y de impacto ambiental, también se verían limitadas por esta condición, lo que se ve reflejado en las reglas de la forma siguiente:

i. Si (Generación es Baja) y (Demanda es Baja), entonces (Costo es Bajo) (Impacto Ambiental es Bajo)(Probabilidad es Alta).

ii. Si (Generación es Baja) y (Demanda es Media), entonces (Costo es Bajo) (Impacto Ambiental es Bajo) (Probabilidad es Media).

iii. Si (Generación es Baja) y (Demanda es Alta), entonces (Costo es Bajo) (Impacto Ambiental es Bajo) (Probabilidad es Media).

iv. $\mathrm{Si}$ (Generación es Media) y (Demanda es Baja), entonces (Costo es Bajo) (Impacto Ambiental es Bajo) (Probabilidad es Media).

v. Si (Generación es Media) y (Demanda es Media), entonces (Costo es Medio) (Impacto Ambiental es Medio) (Probabilidad es Media).

vi. Si (Generación es Media) y (Demanda es Alta), entonces (Costo es Medio) (Impacto Ambiental es Medio)(Probabilidad es Baja).

vii. Si (Generación es Alta) y (Demanda es Baja), entonces (Costo es Bajo) (Impacto Ambiental es Bajo)(Probabilidad es Media).

viii. $\mathrm{Si}$ (Generación es Alta) y (Demanda es Media), entonces (Costo es Medio) (Impacto Ambiental es Medio) (Probabilidad es Baja).

ix. Si (Generación es Alta) y (Demanda es Alta), entonces (Costo es Alto)(Impacto Ambiental es Alto)(Probabilidad es Baja).
Los diagramas de superficie mostrados en la Figura 6 representan el comportamiento de cada variable de salida en función de las variables de entrada para las reglas definidas.

\section{Generación aleatoria de las variables de entrada}

Para establecer los nueve escenarios que representan el conjunto de combinaciones posibles de las variables de entrada, se generaron valores aleatorios de las distribuciones normales de las mismas. Para lograr recorrer todo el rango del potencial se generaron los números aleatorios, utilizando el método de Montecarlo con una distribución normal con los parámetros $\mu$ y $\sigma$ determinados de la manera siguiente:

- Para generar valores bajos de las variables, se utilizó la media y la desviación mostrada en la Tabla 4, la cual se corresponde con el 30\% del potencial.

- Para generar valores medios, se utilizó la media de los valores extremos del potencial y la desviación, lo que se traduce en una traslación de la distribución hacia el centro del rango del potencial.

- Para generar valores altos, se realizó una traslación similar, pero para un $80 \%$ de la suma de los valores extremos y se utilizó la misma desviación.

Se generaron nueve combinaciones de estos valores aleatorios para conformar cada escenario y se evaluaron las variables de entrada en el sistema de inferencia difuso, obteniéndose, para cada par de entrada, la salida correspondiente, como se muestra en la Tabla 6. Con toda la información lista es posible continuar con la aplicación del modelo.
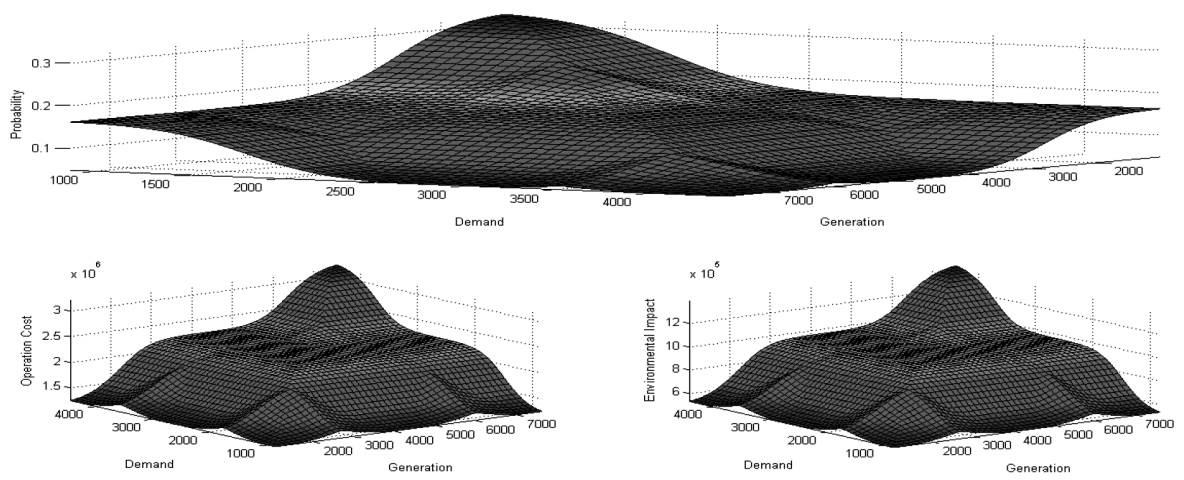

Figura 6. Diagramas de superficie que representan la aplicación de las reglas del sistema de inferencia difusa. Arriba a) Probabilidad, abajo izquierda b) Costos de operaciones y abajo derecha c) Impacto ambiental. 
Tabla 6. Escenarios conformados para la optimización del modelo para el diseño de la cadena de suministros sostenible de plásticos reciclados.

\begin{tabular}{|l|c|c|c|c|c|}
\hline & $\begin{array}{c}\text { Generación } \\
\text { (toneladas) }\end{array}$ & $\begin{array}{c}\text { Demanda } \\
\text { (toneladas) }\end{array}$ & $\begin{array}{c}\text { Costo de operaciones } \\
\text { (Pesos) }\end{array}$ & $\begin{array}{c}\text { Impacto ambiental } \\
\text { (Puntos) }\end{array}$ & Probabilidad \\
\hline Escenario 1 & $1,72 \mathrm{E}+03$ & $1,02 \mathrm{E}+03$ & $1,30 \mathrm{E}+06$ & $5,62 \mathrm{E}+05$ & 0.3108 \\
\hline Escenario 2 & $1,53 \mathrm{E}+03$ & $2,57 \mathrm{E}+03$ & $1,34 \mathrm{E}+06$ & $5,76 \mathrm{E}+05$ & 0.1645 \\
\hline Escenario 3 & $1,75 \mathrm{E}+03$ & $3,74 \mathrm{E}+03$ & $1,51 \mathrm{E}+06$ & $6,51 \mathrm{E}+05$ & 0.1594 \\
\hline Escenario 4 & $4,57 \mathrm{E}+03$ & $9,32 \mathrm{E}+02$ & $1,25 \mathrm{E}+06$ & $5,40 \mathrm{E}+05$ & 0.1641 \\
\hline Escenario 5 & $4,50 \mathrm{E}+03$ & $2,64 \mathrm{E}+03$ & $2,22 \mathrm{E}+06$ & $9,62 \mathrm{E}+05$ & 0.1659 \\
\hline Escenario 6 & $4,09 \mathrm{E}+03$ & $3,74 \mathrm{E}+03$ & $2,22 \mathrm{E}+06$ & $9,62 \mathrm{E}+05$ & 0.0972 \\
\hline Escenario 7 & $7,15 \mathrm{E}+03$ & $9,10 \mathrm{E}+02$ & $1,25 \mathrm{E}+06$ & $5,40 \mathrm{E}+05$ & 0.1588 \\
\hline Escenario 8 & $6,73 \mathrm{E}+03$ & $2,62 \mathrm{E}+03$ & $2,22 \mathrm{E}+06$ & $9,62 \mathrm{E}+05$ & 0.0624 \\
\hline Escenario 9 & $6,73 \mathrm{E}+03$ & $3,63 \mathrm{E}+03$ & $2,57 \mathrm{E}+06$ & $1,12 \mathrm{E}+06$ & 0.0642 \\
\hline
\end{tabular}

\section{Análisis comparativo de las soluciones para todos los escenarios}

Por lo expuesto anteriormente, el modelo desarrollado se optimizó para los nueve escenarios descritos. En este epígrafe se muestran los resultados de todos los escenarios en conjunto, para ello se analizan los potenciales de las soluciones, la función conjunta de los objetivos normalizados y homogenizados.

El potencial de las soluciones se muestra en la Figura 7, donde se pueden apreciar los nueve escenarios y las 25 soluciones de cada uno de ellos. De forma similar a la explicación del epígrafe anterior respecto a este indicador, existen cuatro tipos de soluciones, las cuatro primeras de cada escenario con potencial cero debido a la ausencia de flujos hacia los clientes, las soluciones con potencial de alrededor al $60 \%$, las que tienen este indicador cercano al $80 \%$ con configuraciones de la cadena de suministros similares a la actual y las que mayor nivel de servicio, de aproximadamente el $83 \%$, se corresponden a la cadena propuesta.

En la Figura 7 es notable como en los escenarios donde la demanda tuvo un comportamiento alto y al menos una recuperación media (126-150, 176-200 y 201-225), existen mayor cantidad de soluciones con alto potencial. Estas soluciones no presentan variabilidad en la configuración de la cadena respecto a la propuesta, aunque sí en el comportamiento de los flujos, las cantidades a vender, a recuperar y los inventarios sobrantes. Esto significa que esa configuración es la que mejores posibilidades de nivel de servicio ofrece, independientemente a las cantidades de plásticos reciclables de los tres tipos que se puedan recuperar y vender, sobre todo si estas cantidades tienen comportamientos altos o medios.

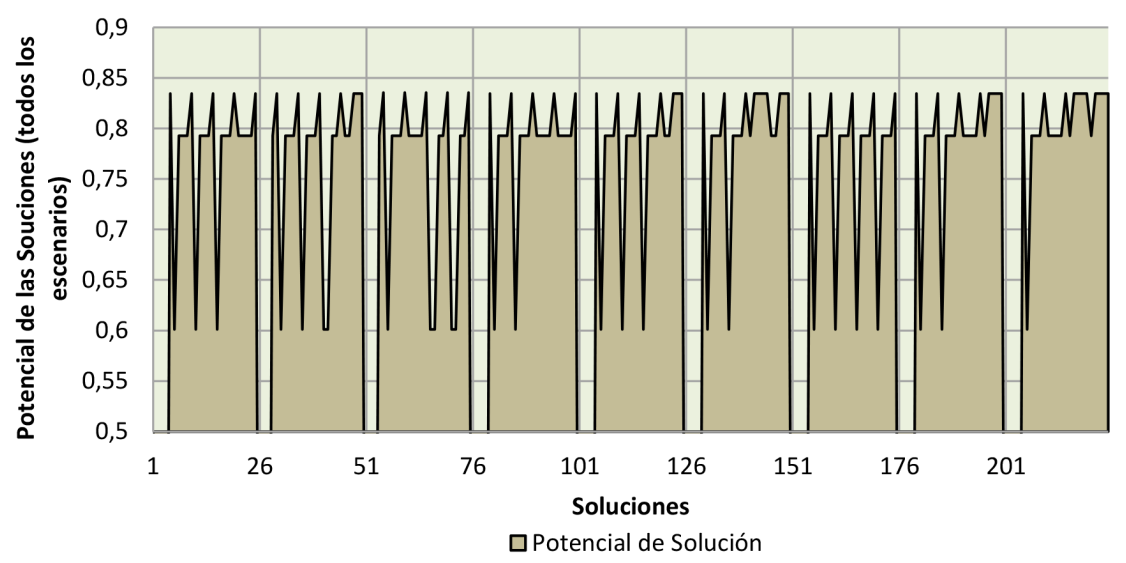

Figura 7. Potencial de las soluciones para todos los escenarios. 
Otra interpretación es que la exploración de los diferentes escenarios no permitió encontrar variabilidad de soluciones con niveles de servicios diferentes. Solamente que a mayores niveles de recuperación y ventas mayor cantidad de soluciones de alto potencial se observan. Esto significa que, para tomar decisiones sobre la localización y la cantidad de plantas de procesamiento de plásticos reciclados y centros recolectores a utilizar, con el fin de encontrar un rediseño de la cadena de suministros sostenible para esta actividad, las soluciones de abrir tres plantas son las mejores para cualquier escenario.

Otro indicador que se analiza es el comportamiento de la sostenibilidad de las soluciones a partir de la función conjunta de los objetivos del modelo, los cuales se muestran en la Figura 8. En esta se puede observar que no hay diferencias significativas en el comportamiento de los objetivos para los diferentes escenarios. Además, se refleja que, a medida que en una solución mejoran los objetivos impacto ambiental y ventas, empeora el costo de operaciones. De lo anterior se concluye, que las soluciones con mejor comportamiento respecto a la sostenibilidad de la cadena de suministro de reciclaje de plásticos, son aquellas que peor costo de operaciones presentan; pero son capaces de maximizar las ventas y el ahorro del impacto ambiental, independientemente de las cantidades de plásticos a reciclar y vender, las cuales se corresponden con la configuración propuesta.

\section{CONCLUSIONES}

El uso de la inferencia difusa para casos de tomas de decisiones que involucren la utilización de expertos o tomadores de decisiones e información es un campo abierto a muchas posibilidades en la gestión empresarial. La utilización del sistema de inferencia difuso constituye un valioso soporte metodológico para la elaboración de escenarios bajo condiciones de incertidumbre y escasez de datos. Este método combina los paradigmas cuantitativos y cualitativos permitiendo integrar el conocimiento de los expertos vinculado con la información estadística disponible, esto conlleva a la conformación de escenarios robustos.

El empleo de escenarios para determinar y probar la factibilidad de las soluciones en diferentes condiciones, permite minimizar el impacto de la incertidumbre en la solución del modelo para el rediseño de la cadena de suministro de reciclaje de plásticos.

Se conformaron un total de nueve escenarios los cuales describen un conjunto exhaustivo de posibilidades suficientemente alto para la generación de un grupo grande de soluciones. Esto posibilita el estudio de una mayor diversidad de configuraciones de la cadena de suministro, así como su desempeño ante las diferentes variaciones de la generación y la demanda.

El análisis de los nueve escenarios permitió validar que la configuración propuesta (abrir tres plantas de producción de plásticos reciclados) es efectiva a las diversas situaciones de generación y demanda posibles en la cadena de suministro para el reciclaje de plásticos en la ERMP.

El valor que tiene la inferencia difusa como herramienta para la toma de decisiones permite aplicaciones muy

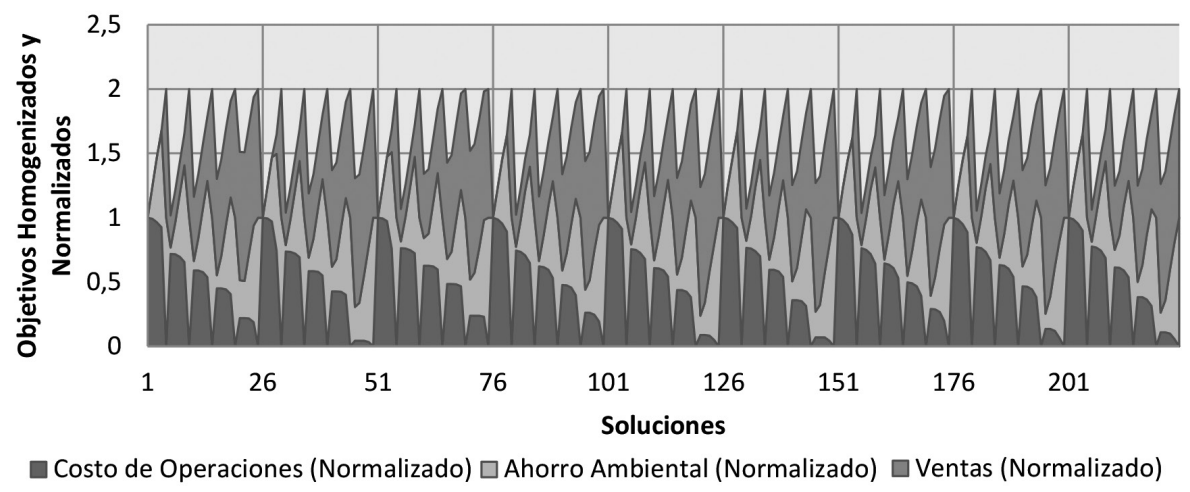

Figura 8. Comportamiento de los objetivos del modelo homogenizados y normalizados para todos los escenarios. 
diversas al campo del control automático donde mayormente se ha desarrollado y además, se perciben oportunidades para su utilización en el pronóstico de la demanda, la gestión de los inventarios, selección de proveedores entre otros aspectos relacionados con la gestión de la cadena de suministros.

\section{REFERENCIAS}

[1] M. Feitó Cespón, R. Cespón Castro, G. Martínez Curbelo y D. Covas Varela. "Diagnóstico ecológico y económico de la cadena de suministros para el reciclaje de plásticos en el contexto empresarial cubano". Estudios Gerenciales. Vol. 31 N $^{\circ} 136$, pp. 347158. 2015. ISSN: 01235923. URL: https:// dx.doi.org/10.1016/j.estger.2015.03.005

[2] S. Pokharel and A. Mutha. "Perspectives in reverse logistics: a review". Resources, Conservation and Recycling. Vol. 53, Issue 4, pp. 175-182. 2009. ISSN: 09213449. URL: https://dx.doi.org/10.1016/j. resconrec.2008.11.006

[3] S. Agrawal, R.K. Singh and Q. Murtaza. "A literature review and perspectives in reverse logistics". Resources, Conservation and Recycling. Vol. 97, pp. 76-92. 2015. ISSN: 18790658. URL: https://dx.doi.org/10.1016/j. resconrec.2015.02.009

[4] K. Govindan, H. Soleimani and D. Kannan. "Reverse logistics and closed-loop supply chain: a comprehensive review to explore the future". European Journal of Operational Research. Vol. 240, Issue 3, pp. 603-626. 2015. ISSN: 0377-2217. URL: https://dx.doi. org/10.1016/j.ejor.2014.07.012

[5] K. Govindan, M. Fattahi and E. Keyvanshokooh. "Supply chain network design under uncertainty: a comprehensive review and future research directions". European Journal of Operational Research. Vol. 263, Issue 1, pp. 108-141. 2017. ISSN: 0377-2217. URL: https://dx.doi.org/10.1016/j. ejor.2017.04.009

[6] M.S. Daskin, C.R. Coullard and Z.-J.M. Shen. "An inventory-location model: formulation, solution algorithm and computational results". Annals of Operations Research. Vol. 110, Issue 1/4, pp. 83-106. 2002. ISSN: 02545330. URL: https://dx.doi. org/10.1023/a:1020763400324
[7] M.C. Georgiadis, P. Tsiakis, P. Longinidis and M.K. Sofioglou. "Optimal design of supply chain networks under uncertain transient demand variations". Omega. Vol. 39, Issue 3, pp. 254-272. 2011. ISSN: 03050483. URL: https://dx.doi.org/10.1016/j. omega.2010.07.002

[8] M. Fattahi and K. Govindan. "Integrated forward/reverse logistics network design under uncertainty with pricing for collection of used products". Annals of Operations Research. Vol. 253, Issue 1, pp. 193-225. 2017. ISSN: 15729338. URL: https://dx.doi. org/10.1007/s10479-016-2347-5

[9] M. Feitó-Cespón, W. Sarache, F. PiedraJimenez and R. Cespón-Castro. "Redesign of a sustainable reverse supply chain under uncertainty: a case study". Journal of Cleaner Production. Vol. 151, pp. 206-217. 2017. ISSN: 0959-6526. URL: https://dx.doi. org/10.1016/j.jclepro.2017.03.057

[10] S.A. Torabi, J. Namdar, S.M. Hatefi and F. Jolai. "An enhanced possibilistic programming approach for reliable closed-loop supply chain network design". International Journal of Production Research. Vol. 54, Issue 5, pp. 1358-1387. 2016. ISSN: 0020-7543. URL: https://dx.doi.org/10.1080/0020754 3.2015.1070215

[11] E. Keyvanshokooh, S.M. Ryan and E. Kabir. "Hybrid robust and stochastic optimization for closed-loop supply chain network design using accelerated benders decomposition". European Journal of Operational Research. Vol. 249, Issue 1, pp. 76-92. 2016. ISSN: 03772217. URL: https://dx.doi.org/10.1016/j. ejor.2015.08.028

[12] M. Brandenburg, K. Govindan, J. Sarkis and S. Seuring. "Quantitative models for sustainable supply chain management: developments and directions". European Journal of Operational Research. Vol. 233, Issue 2, pp. 299-312. 2014. ISSN: 03772217. URL: https://dx.doi.org/10.1016/j. ejor.2013.09.032

[13] H. Soleimani, K. Govindan, H. Saghafi and H. Jafari. "Fuzzy multi-objective sustainable and green closed-loop supply chain network design". Computers \& Industrial Engineering. Vol. 109, pp. 191-203. 2017. ISSN: 0360-8352. URL: https://dx.doi.org/10.1016/j.cie.2017.04.038 
[14] M.S. Pishvaee and M. Fazli Khalaf. "Novel robust fuzzy mathematical programming methods". Applied Mathematical Modelling. Vol. 40, Issue 1, pp. 407-418. 2016. ISSN: 0307-904X. URL: https://dx.doi. org/10.1016/j.apm.2015.04.054

[15] S. Bairamzadeh, M. Saidi-Mehrabad and M.S. Pishvaee. "Modelling different types of uncertainty in biofuel supply network design and planning: a robust optimization approach". Renewable Energy. Vol. 116, pp. 500-517. 2018. ISSN: 09601481. URL: https://dx.doi.org/10.1016/j. renene.2017.09.020

[16] J.C.V. Schmalbach, T.J.F. Herrera, F.J.M. Ávila, J.C. Vergara, T.J. Fontalvo and F. Maza. "La planeación por escenarios: revisión de conceptos y propuestas metodológicas". Prospectiva. Vol. 8, Issue 2, pp. 21-29. 2010. ISSN: 2216-1368

[17] J.M. Latorre, S. Cerisola and A. Ramos. "Clustering algorithms for scenario tree generation: application to natural hydro inflows". European Journal of Operational Research. Vol. 181, Issue 3, pp. 1339-1353. 2007. ISSN: 0377-2217. URL: https://dx.doi. org/10.1016/j.ejor.2005.11.045

[18] N. Gülpınar, B. Rustem and R. Settergren. "Simulation and optimization approaches to scenario tree generation". Journal of Economic Dynamics and Control. Vol. 28, Issue 7, pp. 1291-1315. 2004. ISSN: 01651889. URL: https://dx.doi.org/10.1016/ s0165-1889(03)00113-1

[19] P. Beraldi, F. De Simone and A. Violi. "Generating scenario trees: a parallel integrated simulation-optimization approach". Journal of Computational and Applied Mathematics. Vol. 233, Issue 9, pp. 23222331. 2010. ISSN: 0377-0427. URL: https:// dx.doi.org/10.1016/j.cam.2009.10.017

[20] B.A. Calfa, A. Agarwal, I.E. Grossmann and J.M. Wassick. "Data-driven multi-stage scenario tree generation via statistical property and distribution matching". Computers \& Chemical Engineering. Vol. 68, pp. 7-23. 2014. ISSN: 0098-1354. URL: https://dx.doi. org/10.1016/j.compchemeng.2014.04.012

[21] X. Li, C.L. Ang and R. Gay. "An intelligent scenario generator for strategic business planning”. Computers in Industry. Vol. 34,
Issue 3, pp. 261-269. 1997. ISSN: 01663615. URL: https://dx.doi.org/10.1016/ s0166-3615(97)00062-6

[22] S. Moayer and P.A. Bahri. "Hybrid intelligent scenario generator for business strategic planning by using anfis". Expert Systems with Applications. Vol. 36, Issue 4, pp. 77297737. 2009. ISSN: 09574174. URL: https:// dx.doi.org/10.1016/j.eswa.2008.09.046

[23] M. Dohnal. "Complex biofuels related scenarios generated by qualitative reasoning under severe information shortages: a review". Renewable and Sustainable Energy Reviews. Vol. 65, pp. 676-684. 2016. ISSN: 13640321. URL: https://dx.doi.org/10.1016/j. rser.2016.07.029

[24] A. Ernst, K.H. Biß, H. Shamon, D. Schumann and H.U. Heinrichs. "Benefits and challenges of participatory methods in qualitative energy scenario development". Technological Forecasting and Social Change. Vol. 127, pp. 245-257. 2018. ISSN: 00401625. URL: https://dx.doi.org/10.1016/j. techfore.2017.09.026

[25] L. Melander. "Scenario development in transport studies: methodological considerations and reflections on delphi studies". Futures. Vol. 96, pp. 68-78. 2018. ISSN: 0016-3287. URL: https://dx.doi. org/10.1016/j.futures.2017.11.007

[26] P. Ghadimi, A. Dargi and C. Heavey. "Sustainable supplier performance scoring using audition check-list based fuzzy inference system: a case application in automotive spare part industry". Computers \& Industrial Engineering. Vol. 105, pp. 12-27. 2017. ISSN: 0360-8352. URL: https://dx.doi. org/10.1016/j.cie.2017.01.002

[27] M. Batrouni, A. Bertaux and C. Nicolle. "Scenario analysis, from bigdata to black swan”. Computer Science Review. Vol. 28, pp. 131-139. 2018. ISSN: 15740137. URL: https://dx.doi.org/10.1016/j. cosrev.2018.02.001

[28] G. Wright, G. Cairns, F.A. O'Brien and P. Goodwin. "Scenario analysis to support decision making in addressing wicked problems: pitfalls and potential". European Journal of Operational Research. 2018. ISSN: 03772217. URL: https://dx.doi.org/10.1016/j. ejor.2018.08.035 
[29] G. Ringland and P. Préfacier "SCHWARTZ" Scenario planning: managing for the future "Wiley". ISBN: 047197790X. 1998.

[30] A.S. Oliveira, M.D. de Barros, F. de Carvalho Pereira, C.F.S. Gomes and H.G. da Costa. "Prospective scenarios: a literature review on the scopus database". Futures. Vol. 100, pp. 20-33. 2018. ISSN: 00163287. URL: https://dx.doi.org/10.1016/j. futures.2018.03.005

[31] G. Guastaroba, R. Mansini and M.G. Speranza. "On the effectiveness of scenario generation techniques in single-period portfolio optimization". European Journal of Operational Research. Vol. 192, Issue 2, pp. 500-511. 2009. ISSN: 0377-2217. URL: https://dx.doi.org/10.1016/j.ejor.2007.09.042

[32] Y. Tokat, S.T. Rachev and E.S. Schwartz. "The stable non-gaussian asset allocation: a comparison with the classical gaussian approach". High-Performance Computing for Financial Planning. Vol. 27, Issue6, pp. 937-969. 2003. ISSN: 0165-1889. URL: https://dx.doi.org/10.1016/ s0165-1889(02)00050-7

[33] F.L.C. Oliveira, R.C. Souza and A.L.M. Marcato. "A time series model for building scenarios trees applied to stochastic optimisation". International Journal of Electrical Power \& Energy Systems. Vol. 67, pp. 315-323. 2015. ISSN: 0142-0615. URL: https://doi.org/10.1016/j.ijepes.2014.11.031

[34] B. Jiao, C. Wang and L. Guo. "Scenario generation for energy storage system design in stand-alone microgrids". International Conference on Applied Energy, ICAE2014. Vol. 61, pp. 824-828. 2014. ISSN: 18766102. URL: https://dx.doi.org/10.1016/j. egypro.2014.11.974

[35] S. Araghi, A. Khosravi, D. Creighton and S. Nahavandi. "Influence of meta-heuristic optimization on the performance of adaptive interval type2-fuzzy traffic signal controllers". Expert Systems with Applications. Vol. 71, pp. 493-503. 2017. ISSN: 0957-4174. URL: https://dx.doi.org/10.1016/j.eswa.2016.10.066

[36] M. Düspohl and P. Döll. "Causal networks and scenarios: participatory strategy development for promoting renewable electricity generation". Journal of Cleaner Production. Vol. 121, pp. 218-230. 2016.
ISSN: 0959-6526. URL: https://dx.doi. org/10.1016/j.jclepro.2015.09.117

[37] A.J. Symstad, N.A. Fisichelli, B.W. Miller, E. Rowland and G.W. Schuurman. "Multiple methods for multiple futures: integrating qualitative scenario planning and quantitative simulation modeling for natural resource decision making". Climate Risk Management. Vol. 17, pp. 78-91. 2017. ISSN: 2212 0963. URL: https://dx.doi.org/10.1016/j. crm.2017.07.002

[38] M. Sotirov, M. Blum, S. Storch, A. Selter and U. Schraml. "Do forest policy actors learn through forward-thinking? conflict and cooperation relating to the past, present and futures of sustainable forest management in germany". Forest Policy and Economics. Vol. 85, pp. 256-268. 2017. ISSN: 13899341. URL: https://dx.doi.org/10.1016/j. forpol.2016.11.011

[39] Y. Hasegawa, K. Okabe and H. Taki. "A scenario approach for ecosystem-service changes". Futures. Vol. 96, pp. 23-31. 2018. ISSN: 00163287. URL: https://dx.doi. org/10.1016/j.futures.2017.11.003

[40] S. Di Zio, J.D. Castillo Rosas and L. Lamelza. "Real time spatial delphi: fast convergence of experts' opinions on the territory". Technological Forecasting and Social Change. Vol. 115, pp. 143-154. 2017. ISSN: 00401625. URL: https://dx.doi. org/10.1016/j.techfore.2016.09.029

[41] J. Carbonell, A. Sánchez-Esguevillas and B. Carro. "From data analysis to storytelling in scenario building. a semiotic approach to purpose-dependent writing of stories". Futures. Vol. 88, pp. 15-29. 2017. ISSN: 00163287. URL: https://dx.doi.org/10.1016/j. futures.2017.03.002

[42] K.L. Lam, J.R. Stokes-Draut, A. Horvath, J.L. Lane, S.J. Kenway and P.A. Lant. "Lifecycle energy impacts for adapting an urban water supply system to droughts". Water Research. Vol. 127, pp. 139-149. 2017. ISSN: 18792448. URL: https://dx.doi.org/10.1016/j. watres.2017.10.016

[43] R.M. Deus, R.A.G. Battistelle and G.H.R. Silva. "Current and future environmental impact of household solid waste management scenarios for a region of brazil: carbon dioxide and energy analysis". Journal of 
Cleaner Production. Vol. 155, pp. 218-228. 2017. ISSN: 09596526. URL: https://dx.doi. org/10.1016/j.jclepro.2016.05.158

[44] R. Bourgeois, E. Penunia, S. Bisht and D. Boruk. "Foresight for all: co-elaborative scenario building and empowerment". Technological Forecasting and Social Change. Vol. 124, pp. 178-188. 2017. ISSN: 00401625. URL: https://dx.doi.org/10.1016/j. techfore.2017.04.018

[45] P.S. Andersen, E. Andersen, M. Graversgaard, A.A. Christensen, H. Vejre and T. Dalgaard. "Using landscape scenarios to improve local nitrogen management and planning". Journal of Environmental Management. Vol. 232, pp. 523530. 2019. ISSN: 10958630. URL: https:// dx.doi.org/10.1016/j.jenvman.2018.11.023

[46] N. Maestripieri, T. Houet, M. Paegelow, G. Selleron, D. Toro Balbontín and N. Sáez Villalobos. "Dynamic simulation of forest management normative scenarios: the case of timber plantations in the southern chile". Futures. Vol. 87, pp. 65-77. 2017. ISSN: 00163287. URL: https://dx.doi.org/10.1016/j. futures.2015.10.013

[47] M. Martín-Gamboa, D. Iribarren, D. García-Gusano and J. Dufour. "Enhanced prioritisation of prospective scenarios for power generation in spain: how and which one?". Energy. Vol. 169, pp. 369-379. 2019. ISSN: 03605442

[48] N. Anh, M. Prasad, N. Srikanth and S. Sundaram. "Wave forecasting using metacognitive interval type-2 fuzzy inference system". Procedia Computer Science. Vol. 144, pp. 33-41. 2018. ISSN: 18770509. URL: https://dx.doi.org/10.1016/j.procs.2018.10.502.

[49] M. Dehghanbaghi, H. Hosseininasab and A. Sadeghieh. "A hybrid approach to support recovery strategies (a case study)". Journal of Cleaner Production. Vol. 113, pp. 717729. 2016. ISSN: 0959-6526. URL: https:// dx.doi.org/10.1016/j.jclepro.2015.11.064

[50] A.A.M. Ahmed and S.M.A. Shah. "Application of adaptive neuro-fuzzy inference system (anfis) to estimate the biochemical oxygen demand (bod) of surma river". Journal of King Saud University - Engineering Sciences. Vol. 29, Issue 3, pp. 237-243. 2017. ISSN: 10183639. URL: https://dx.doi.org/10.1016/j. jksues.2015.02.001
[51] I. Pluchinotta, D. Esposito and D. Camarda. "Fuzzy cognitive mapping to support multiagent decisions in development of urban policymaking". Sustainable Cities and Society. Vol. 46, p. 101402. 2019. ISSN: 22106707. URL: https://dx.doi.org/10.1016/j. scs.2018.12.030

[52] M. Feitó Cespón, R. Cespón Castro y M.A. Rubio Rodríguez. "Modelos de optimización para el diseño sostenible de cadenas de suministros de reciclaje de múltiples productos". Ingeniare. Revista chilena de ingeniería. Vol. 24 $\mathrm{N}^{\mathrm{o}}$ 1, pp. 135-148. 2016. ISSN: 0718-3305

[53] V. De Rosa, M. Gebhard, E. Hartmann and J. Wollenweber. "Robust sustainable bi-directional logistics network design under uncertainty". International Journal of Production Economics. Vol. 145, Issue 1, pp. 184-198. 2013. ISSN: 0925-5273. URL: https://dx.doi.org/10.1016/j.ijpe.2013.04.033

[54] M.S. Pishvaee, M. Rabbani and S.A. Torabi. "A robust optimization approach to closed-loop supply chain network design under uncertainty". Applied Mathematical Modelling. Vol. 35, Issue 2, pp. 637-649. 2011. ISSN: 0307-904X. URL: https:// dx.doi.org/10.1016/j.apm.2010.07.013

[55] S.M. Hatefi and F. Jolai. "Robust and reliable forward-reverse logistics network design under demand uncertainty and facility disruptions". Applied Mathematical Modelling. Vol. 38, Issue 9-10, pp. 26302647. 2014. ISSN: 0307-904X. URL: https:// dx.doi.org/10.1016/j.apm.2013.11.002

[56] R.H. Ballou "Business logistics management: planning, organizing, and controlling the supply chain; instructorś manual". PrenticeHall. ISBN: 0130807133. 1999.

[57] M. Ramezani, A.M. Kimiagari, B. Karimi and T.H. Hejazi. "Closed-loop supply chain network design under a fuzzy environment". Knowledge-Based Systems. Vol. 59, Issue 0, pp. 108-120. 2014. ISSN: 09507051. URL: https://dx.doi.org/10.1016/j. knosys.2014.01.016

[58] E.H. Mamdani and S. Assilian. "An experiment in linguistic synthesis with a fuzzy logic controller". International Journal of ManMachine Studies. Vol. 7, Issue 1, pp. 1-13. 1975. ISSN: 0020-7373. URL: https://dx.doi. org/10.1016/s0020-7373(75)80002-2 
[59] E.H. Mamdani. "Application of fuzzy logic to approximate reasoning using linguistic synthesis". Computers, IEEE Transactions on. Vol. C-26, Issue 12, pp. 1182-1191. 1977. ISSN: 0018-9340. URL: https://dx.doi. org/10.1109/tc.1977.1674779
[60] L.A. Zadeh. "Outline of a new approach to the analysis of complex systems and decision processes". Systems, Man and Cybernetics, IEEE Transactions on. Vol. SMC-3, Issue 1, pp. 28-44. 1973. ISSN: 0018-9472. URL: https://dx.doi.org/10.1109/tsmc.1973.5408575

Anexo 1.Modelo multiobjetivo para el rediseño de la CS sostenible de reciclaje múltiples productos. Tomado de Feitó-Cespón, Sarache, Piedra-Jimenez y Cespón-Castro (2017) [9]

\section{Conjuntos}

I: conjunto de locales de proveedores generadores de residuos reciclables $(i=1,2, \ldots, \mathrm{I})$.

$\mathrm{J}$ : conjunto de locales potenciales de centros de recolección $(\mathrm{j}=1,2, \ldots, \mathrm{J})$.

$\mathrm{K}$ : conjunto de plantas de procesamiento potenciales $(\mathrm{k}=1,2, \ldots, \mathrm{K})$.

L: conjunto de clientes $(1=1,2, \ldots, \mathrm{L})$.

M: conjunto de medios de transporte potenciales $(\mathrm{m}=1,2, \ldots, \mathrm{M})$.

$\mathrm{P}$ : conjunto de productos a reciclar $(\mathrm{p}=1,2, \ldots, \mathrm{P})$.

\section{Variables de decisión}

$Q S R_{i j m p}$ : cantidad de producto $\mathrm{p}$ a transportar en el medio m, entre proveedor i y el centro de recolección j. $Q R P_{j k m p}$ : cantidad de producto p a transportar en el medio m, entre el centro de recolección j y la planta k. $Q P C_{k l m p}$ : cantidad de producto $\mathrm{p}$ a transportar el medio m, entre la planta $\mathrm{k}$ y el cliente $\mathrm{l}$.

$V S R_{i j m}$ : cantidad de viajes entre el suministrador i y el centro de recolección j utilizando el medio $\mathrm{m}$. $V R P_{j k m}$ : cantidad de viajes entre el centro de recolección j y la planta k utilizando el medio $\mathrm{m}$. $V P C_{k l m}$ : cantidad de viajes entre la planta de producción $\mathrm{k}$ y el cliente 1 utilizando el medio $\mathrm{m}$. $H S R_{i j m}, H R P_{j k m}, H P C_{k l m}$ : son variables para representar las cantidades de viajes por exceso y defecto, necesarias para el balance de los medios de transporte.

Rj (utilización del centro de recolección j) y Pk (utilización de la planta k), constituyen variables binarias que se definen como sigue:

$$
\begin{array}{r}
D C_{i j}=\left\{\begin{array}{rr}
1, & \text { Si } f_{1 \mathrm{ij}} \varphi_{1 \mathrm{j}}, \\
0, & \text { de cualquier otra forma. }
\end{array}\right. \\
D A_{i j}=\left\{\begin{array}{rr}
1, & \text { Si } f_{2 \mathrm{ij}} \varphi_{2 \mathrm{j}}, \\
0, & \text { de cualquier otra forma. }
\end{array}\right.
\end{array}
$$

\section{Parámetros}

$G_{i p}$ : cantidad de producto p del suministrador i generado en el período.

$C T_{m p}$ : capacidad del medio de transporte m para transportar el producto $\mathrm{p}$.

$C_{k p}$ : capacidad de la planta k para procesar el producto $\mathrm{p}$ en el período.

$D_{l p}$ : demanda de cada cliente 1 del producto $\mathrm{p}$ en el período.

$N_{v m}$ : cantidad de viajes disponibles en el período para cada medio de transporte m.

$I_{p}$ : impacto ecológico por producir una unidad nueva del producto $\mathrm{p}$.

$I T_{m}$ : impacto ecológico por transportar una tonelada-kilómetro en el medio de transporte $\mathrm{m}$.

$I E$ : impacto ecológico por consumir un kilowatt hora en el sistema.

$I P$ : impacto ecológico producido por la infraestructura.

IA: impacto ecológico por consumir un kilogramo de agua en el sistema.

$I V_{p}$ : impacto ecológico por desechar en un vertedero una unidad del producto $\mathrm{p}$, luego de su consumo. 
$C f_{e k}$ : consumo eléctrico fijo de la planta de reciclaje $\mathrm{k}$ (depende de los equipos consumidores eléctricos que no son directos a la producción, necesarios para operar la planta).

$C f_{e i}$ : consumo eléctrico fijo del centro recolector $\mathrm{j}$.

$C v_{e p}$ : consumo variable de electricidad necesaria para procesar una unidad del producto $\mathrm{p}$.

$C v_{a p}$ : consumo variable de agua necesaria para procesar una unidad del producto $\mathrm{p}$.

$a_{k}$ y $\beta_{j}$ : proporción de la capacidad de la planta de reciclaje $\mathrm{k}$ y el centro recolector $\mathrm{j}$ respectivamente, de la planta de reciclaje tipo registrada en la base de datos Ecoinvent.

$d_{i j}^{S R}:$ distancias entre el suministrador i y cada centro de recolección j.

$d_{j k}^{R P}$ : distancias entre el centro de recolección j y cada planta de procesamiento $\mathrm{k}$.

$d_{k l}^{P G}$ : distancias entre la planta k y cada cliente 1 .

$C U R_{j p}$ : costo unitario del producto $\mathrm{p}$ en el centro de recolección $\mathrm{j}$, en pesos/unidad.

$C U P_{k p}$ : costo unitario del producto $\mathrm{p}$ en la planta k, en pesos/unidad.

$C U T_{m}$ : costo variable por tkm del medio de transporte $\mathrm{m}$, en pesos/tkm.

$C F R_{j}$ : costo fijo por utilizar el centro de recolección $\mathrm{j}$, en pesos/período.

$C F P_{k}$ : costo fijo por utilizar la planta k, en pesos/período.

$C F T_{m}$ : costo fijo por utilizar el medio de transporte m, en un viaje en pesos/viaje.

$$
\begin{aligned}
& \min f_{1} \\
& =\sum_{m} C F T_{m}\left(\sum_{i} \sum_{j} V S R_{i j m}+\sum_{j} \sum_{k} V R P_{j k m}+\sum_{k} \sum_{l} V P C_{k l m}\right) \\
& +\sum_{m} C U T_{m}\left(\sum_{i} \sum_{j}\left(\sum_{p} Q S R_{i j m p}\right) V S R_{i j m} d_{i j}^{S R}+\sum_{j} \sum_{k}\left(\sum_{p} Q R P_{j k m p}\right) V R P_{j k m} d_{j k}^{R P}\right. \\
& \left.+\sum_{k} \sum_{l}\left(\sum_{p} Q P C_{k l m p}\right) V P C_{k l m} d_{k l}^{P C}\right)+\sum_{k} C F P_{k} P_{k}+\sum_{k} \sum_{p} C U P_{k p} \sum_{l} \sum_{m} Q P C_{k l m p} \\
& +\sum_{j} C F R_{j} R_{j}+\sum_{j} \sum_{p} C U R_{j p} \sum_{k} \sum_{m} Q R P_{j k m p} \\
& \max _{2}=\sum_{p} I_{p} \sum_{k} \sum_{l} \sum_{m} Q P C_{k l m p} \\
& -\left[I T \left(\sum_{i} \sum_{j} \sum_{m} V S R_{i j m} d_{i j}^{S R} \sum_{i} \sum_{j} \sum_{m} \sum_{p} Q S R_{i j m p}\right.\right. \\
& +\sum_{j} \sum_{k} \sum_{m} V R P_{j k m} d_{j k}^{R P} \sum_{i} \sum_{j} \sum_{m} \sum_{p} Q R P_{j k m p} \\
& \left.+\sum_{k} \sum_{l} \sum_{m} V P C_{k l m} d_{k l}^{P C} \sum_{i} \sum_{j} \sum_{m} \sum_{p b} Q P C_{k l m p}\right) \\
& +I E\left(\sum_{k} C f e_{k} P_{k}+\sum_{j} C f e_{j} R_{j}+\sum_{p} C v e_{p} \sum_{k} \sum_{l} \sum_{m} Q P C_{k l m p}\right) \\
& \left.+I P\left(\sum_{k} \alpha_{k} P_{k}+\sum_{j} \beta_{j} R_{j}\right)+I A \sum_{p} C v a_{p} \sum_{k} \sum_{l} \sum_{m} Q P C_{k l m p}\right] \\
& +\sum_{p} I V_{p} \sum_{i} \sum_{i} \sum_{m} Q S R_{i j m p}
\end{aligned}
$$


Sujeto a

$$
\max f_{3}=\sum_{k} \sum_{l} \sum_{m} \sum_{p} Q P C_{k l m p}
$$

$$
\begin{array}{cl}
\sum_{j} \sum_{m} Q S R_{i j m p} \leq G_{i p}, & \forall i, p \\
\sum_{k} \sum_{m}^{m} Q R P_{j k m p} \leq \sum_{i} \sum_{m}^{m} Q S R_{i j m p}, & \forall j, p \\
\sum_{l} \sum_{m} Q P C_{k l m p} \leq \sum_{j} \sum_{m} Q R P_{j k m p}, & \forall k, p \\
\sum_{l} \sum_{m}^{m} Q P C_{k l m p} \leq C_{k p}, & \forall k, p \\
\sum_{k} \sum_{m} Q P C_{k l m p} \leq D_{l p}, & \forall l, p \\
\sum_{p} Q R R_{i j m p} / C T_{m p}+H S R_{i j m}=V S R_{i j m}, & \forall i, j, m \\
Q P C_{k l m p} / C T_{m p}+H P C_{k l m}=V P C_{k l m}, & \forall k, l, m \\
V S R_{i j m}+H S R_{i j m} \geq 0, \quad \forall i, j, m & \forall j, k, m \\
V R P_{j k m}+H R P_{j k m} \geq 0, \quad \forall j, k, m &
\end{array}
$$

Al final del modelo es necesario establecer los límites de las variables: todas las variables que representan flujos son continuas y mayores o iguales que 0 , las que representan el número de viajes entre un punto y otro son enteras, también mayores o iguales a 0 , las variables que constituyen las holguras son continuas, mayores que -1 y menores que 1 y las binarias son enteras, mayor o igual a 0 y menor o igual que 1 . 